\title{
Visceral adipose NLRP3 impairs cognition in obesity via IL-1R1 on CX3CR1 ${ }^{+}$cells
}

\author{
De-Huang Guo, ${ }^{1}$ Masaki Yamamoto, ${ }^{1}$ Caterina M. Hernandez, ${ }^{2}$ Hesam Khodadadi, ${ }^{3}$ Babak Baban, ${ }^{3,4}$ and Alexis M. Stranahan ${ }^{1}$ \\ DDepartment of Neuroscience and Regenerative Medicine, ${ }^{2}$ Department of Pharmacology and Toxicology, ${ }^{3}$ Department of Oral Biology, and ${ }^{4}$ Plastic Surgery Section, Department of Surgery, Medical College of \\ Georgia, Augusta University, Augusta, Ceorgia, USA.
}

\begin{abstract}
Induction of the inflammasome protein cryopyrin (NLRP3) in visceral adipose tissue (VAT) promotes release of the proinflammatory cytokine IL-1 $1 \beta$ in obesity. Although this mechanism contributes to peripheral metabolic dysfunction, effects on the brain remain unexplored. We investigated whether visceral adipose NLRP3 impairs cognition by activating microglial IL-1 receptor 1 (IL-1R1). After observing protection against obesity-induced neuroinflammation and cognitive impairment in NLRP3-KO mice, we transplanted VAT from obese WT or NLRP3-KO donors into lean recipient mice. Transplantation of VAT

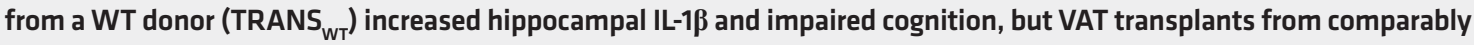
obese NLRP3-KO donors (TRANS ${ }_{\text {Ко }}$ ) had no effect. Visceral adipose NLRP3 was required for deficits in long-term potentiation (LTP) in transplant recipients, and LTP impairment in TRANS ${ }_{\mathrm{WT}}$ mice was IL-1 dependent. Flow cytometric and gene expression analyses revealed that VAT transplantation recapitulated the effects of obesity on microglial activation and IL-1 $\beta$ gene expression, and visualization of hippocampal microglia revealed similar effects in vivo. Inducible ablation of IL-1R1 in CX3CR1expressing cells eliminated cognitive impairment in mice with dietary obesity and in transplant recipients and restored immunoquiescence in hippocampal microglia. These results indicate that visceral adipose NLRP3 impairs memory via IL-1mediated microglial activation and suggest that NLRP3/IL-1及 signaling may underlie correlations between visceral adiposity and cognitive impairment in humans.
\end{abstract}

\section{Introduction}

Obesity is a major public health issue, and although some overweight individuals are physiologically healthy, many develop serious pathological conditions. Obesity has a deleterious effect on the CNS, and these effects are not limited to classical metabolic circuits in the brain. Several studies have reported increased rates of age-related cognitive decline in human obesity (1-5) and atrophy of medial temporal lobe regions involved in memory, including the hippocampus (6-8). However, there are also negative reports that did not detect cognitive impairment or brain atrophy in obesity (9-11). Unresolved controversies surrounding obesity-induced cognitive impairment may be due to the use of weight/height ratio criteria that do not reflect differences in adipose tissue distribution. Individuals with the "apple-shaped" distribution of body fat are at increased risk of developing diabetes (12). Visceral adiposity, as reflected by the waist/hip ratio, is a stronger predictor of age-related cognitive impairment than is BMI in humans $(4,13,14)$. Although consensus is emerging from human studies of dementia risk in obesity, work in animal models has yet to elucidate the specific mechanism linking visceral adiposity with cognitive impairment.

Deposition of visceral adipose tissue (VAT) induces systemic inflammation and promotes the development of metabolic com-

Conflict of interest: The authors have declared that no conflict of interest exists. Copyright: () 2020, American Society for Clinical Investigation.

Submitted: November 7, 2018; Accepted: January 3, 2020; Published: March 9, 2020.

Reference information: J Clin Invest. 2020;130(4):1961-1976.

https://doi.org/10.1172/JCI126078. plications in obesity $(15,16)$. Chronic lipid overload in visceral adipocytes is accompanied by the release of damage-associated molecular patterns that attract monocyte precursors and induce their differentiation into adipose tissue macrophages (17). The inflammatory VAT microenvironment leads to the formation of inflammasome complexes that amplify innate immune responses in adipose tissue $(18,19)$. NOD-like receptor family, pyrin domain-containing 3 (NLRP3) is a core component of the inflammasome complex, and visceral adipose NLRP3 induction promotes synthesis and release of the proinflammatory cytokine IL-1 $\beta$ in obesity (18). Whole-body $\mathrm{Nlrp}^{-/-}$mice develop obesity but are protected against high-fat diet-induced (HFD-induced) adipose tissue inflammation and insulin resistance $(18,19)$. The Nlrp3-null mutation also protects against cognitive deficits in aged mice and in mouse models of Alzheimer's disease $(20,21)$, but the consequences of tissue-specific NLRP3 induction for neuroplasticity and neuroinflammation have yet to be elucidated.

We hypothesized that NLRP3 induction in VAT initiates microglial activation and cognitive impairment by increasing IL-1 $\beta$. Peripheral IL-1 $\beta$ enters the CNS via saturable transporters at the blood-brain barrier (BBB) (22), and we reasoned that microglial IL-1 receptor 1 (Illr1) activation would initiate an autocrine amplification loop similar to that previously reported in other disease models (23). This hypothesis was tested in a series of dietary obesity and VAT transplantation experiments using $\mathrm{Nlrp3}^{-/-}$mutant mice and Tg mice with inducible deletion of Illr1 in CX3CR1-expressing cells. Collectively, these data suggest that CNS immune cells detect and amplify peripheral IL- $1 \beta$ generated 
A



B



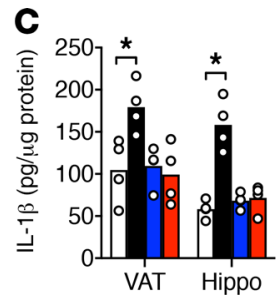

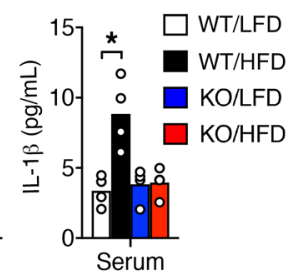



E

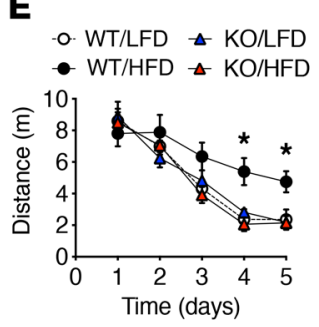



IBA1/CD68/DAP|


Figure 1. Protection against obesity-induced hippocampal dysfunction in mice lacking the inflammasome protein NLRP3. (A) Male WT and NLRP3-KO mice gained similar amounts of weight over time $(n=20)$. (B) Adipose tissue hypertrophy was comparable in WT/HFD and KO/HFD mice $(n=6)$. BAT, brown adipose tissue. (C) KO/HFD mice were protected against increases in IL-1 $\beta$ in VAT, hippocampus (Hippo), and serum $(n=4)$. (D) NLRP3-dependent reductions in microglial process complexity and total length with dietary obesity $(n=4)$. Micrographs show increased CD68 immunoreactivity in WT but not KO/HFD mice (see also Supplemental Figure 1). Scale bar: $10 \mu \mathrm{m}$. (E) NLRP3 was required for obesity-induced memory impairment in the water maze during acquisition training and the probe test $(n=16)$. (F) Dentate gyrus LTP was significantly reduced in an NLRP3-dependent manner with dietary obesity $(n=10-12$ slices, $n=4-6$ mice). For traces (inset, left), scale bars: $x=1 \mathrm{msec}, \mathrm{y}=1 \mathrm{mV}$. Data indicate the mean $\pm \mathrm{SEM}$. ${ }^{*} P<0.05$, by 2 -way repeated-measures ANOVA (A) or 2-way ANOVA (B-F) with Tukey's HSD post hoc test.

following visceral adipose inflammasome activation and that increase in CNS IL-1 $\beta$ downstream of this cascade impair hippocampal synaptic plasticity and cognition in obesity. Our findings add to the growing literature on dynamic interactions between the brain and peripheral tissues and provide further support for reinterpretation of immune privilege in the CNS.

\section{Results}

Resistance to obesity-induced neuroinflammation and cognitive dysfunction in NLRP3-KO mice. Obesity promotes the formation of inflammasome complexes in multiple tissues, including the brain (24), but the link between NLRP3 and obesity-induced cognitive impairment remains correlative at present. To determine whether the inflammasome protein NLRP3 is required for obesity-induced microglial activation and cognitive dysfunction, we maintained $N \operatorname{lrp3^{-/-}}$ (KO) mice and WT littermates on either a HFD or a lowfat diet (LFD). Consistent with previous reports $(18,19)$, WT and NLRP3-KO mice gained comparable amounts of weight during the 12-week period (Figure 1A). The weight of the visceral, subcutaneous, and interscapular fat pads was also unaffected by genotype (Figure 1B). However, NLRP3-KO mice were protected against increases in IL-1 $\beta$ in VAT with HFD consumption (Figure $1 \mathrm{C} ; \mathrm{F}_{1 / 12}$ $=16.83, P=0.002)$. HFD-fed WT mice (WT/HFD mice) exhibited significant increases in circulating IL- $1 \beta\left(\mathrm{F}_{1 / 12}=8.05, P=0.02\right)$, but levels in HFD-fed NLRP3-KO mice (NLRP3-KO/HFD mice) did not differ from those in LFD-fed WT mice (WT/LFD mice) (Figure 1C). Protection against IL-1 $\beta$ accumulation was also observed in hippocampal lysates from NLRP3-KO/HFD mice (Figure 1C; $\mathrm{F}_{1 / 12}=6.8, P=0.02$ ), suggesting that resistance to obesity-induced peripheral inflammation in NLRP3-KO mice extends to the CNS.

Microglia continuously sense and respond to molecular patterns in the local environment. Because microglial process retraction is a well-characterized response to inflammation, we examined IBA $^{+}$cells in the hippocampal dentate gyrus and analyzed their morphology. Microglia from WT/HFD mice showed significant reductions in process length and complexity, as indicated by lower numbers of intersections at 1-micron intervals around the soma (Figure 1D, $\mathrm{F}_{1,14}=6.61, P=0.02$ ). Process length and complexity were unaffected in NLRP3-KO/HFD mice, which did not differ from WT/LFD mice in this regard (Figure 1D). We detected no differences between WT/LFD and NLRP3-KO/LFD mice, indicating that NLRP3-KO mice were resistant to HFD-induced microglial process retraction. Parallel examination of the lysosomal marker CD68 revealed significant accumulation in induction of brown adipocytes 1-positive (IBA1 ${ }^{+}$) microglia in WT/HFD mice, but not in NLRP3-KO/HFD mice (Figure 1D). Microglial CD68 accumulation was evident both qualitatively and quantitatively, as determined by analysis of $\mathrm{CD} 68^{+}$puncta within regions of interest delineated by IBA1 labeling (Supplemental Figure 1, A and B; $F_{1,14}=13.47, P<$ 0.001 ; supplemental material available online with this article; 
https://doi.org/10.1172/JCI126078DS1). Microglial CD68 accumulation occurs following phagocytosis, which could reflect protective or pathological responses (25). To interpret changes in CD68 immunoreactivity, we performed immunofluorescence labeling for IBA1 and the classical activation marker MHC class II (MHCII). Consistent with previous studies (26), IBA1-MHCII-doublepositive cells were more frequent in WT/HFD mice relative to WT/ LFD (Supplemental Figure 1, C and D; $\mathrm{F}_{1,14}=17.84, P<0.001$ ). Colabeling for IBA1 and MHCII was rare in NLRP3-KO/HFD mice, as was observed in WT/LFD mice (Supplemental Figure 1, C and D).

To examine the role of NLRP3 in obesity-induced cognitive dysfunction, WT and NLRP3-KO mice were tested in the water maze after 12 weeks of a HFD or a LFD. WT/HFD mice had longer swimming paths during acquisition training and exhibited deficits during the probe test relative to WT/LFD mice (Figure 1E; for acquisition, $\mathrm{F}_{1,36}=7.03, P=0.01$; for probe, $\mathrm{F}_{1,60}=4.11, P=0.04$ ). By contrast, the swimming distance for NLRP3-KO/HFD mice did not differ from that of WT/LFD mice (Figure 1E). There was no effect of genotype in LFD mice, and all groups of mice performed similarly when swimming toward a visible platform (distance $[\mathrm{m}]$, mean \pm SEM: WT/LFD $=5.8 \pm 1.1 ; \mathrm{WT} / \mathrm{HFD}=5.9 \pm 0.7 ; \mathrm{KO} / \mathrm{LFD}$ $=5.4 \pm 0.8 ; \mathrm{KO} / \mathrm{HFD}=5.3 \pm 1.0)$. After observing NLRP3-dependent deficits in learning and memory, we investigated changes in hippocampal synaptic plasticity using extracellular recordings in brain slices. Stimulation of medial perforant path afferents to the dentate gyrus revealed significant reductions in long-term potentiation (LTP) in WT/HFD mice (Figure $1 \mathrm{~F} ; \mathrm{F}_{1,39}=6.88, P=0.01$ ). Deficits in LTP were mediated by NLRP3, as slices from NLRP3KO/HFD mice had LTP that was comparable to that of WT/LFD (Figure $1 \mathrm{~F}$ ), indicating that whole-body ablation of NLRP3 protects against obesity-induced microglial activation and maintains hippocampal plasticity in dietary obesity.

Visceral adipose NLRP3 increases hippocampal IL-1 $\beta$. To determine whether NLRP3 induction in visceral fat regulates hippocampal IL-1 $\beta$, we transplanted VAT from WT/HFD or NLRP3$\mathrm{KO} / \mathrm{HFD}$ donors into lean WT recipients (Figure 2A). Mice that received transplants from a WT donor TRANS $_{\mathrm{WT}}$ ) or an NLRP3KO donor (TRANS $_{\mathrm{KO}}$ ) were compared with sham-operated WT mice maintained on a LFD (LFD/SHAM) or a HFD (HFD/SHAM). Visceral fat transplantation had no effect on body weight or glycemic control in recipient mice 2 weeks after surgery (Figure 2, $\mathrm{A}$ and $\mathrm{B}$ ), and there were no differences in transplant viability between WT and NLRP3-KO donors (Supplemental Figure 2A; number of rejections: TRANS ${ }_{\mathrm{WT}}, n=1$; $\left.^{\text {TRANS }}{ }_{\mathrm{KO}}, n=2\right)$. We also found no evidence of compensatory atrophy in resident fat pads from transplant recipients (Supplemental Figure 2B). The cleavage and release of IL-1 $\beta$ is a prominent consequence of visceral adipose NLRP3 induction (18). Quantification of IL-1 $\beta$ in hippocampus, VAT, and serum revealed parallel increases in TRANS and HFD/SHAM mice (Figure 2C). Increases in hippocampal IL-1 $\beta$ were dependent on NLRP3, as TRANS ${ }_{\mathrm{KO}}$ mice had lower levels of IL-1 $\beta$ than did TRANS ${ }_{\mathrm{WT}}$ mice, and these levels did not differ from those in LFD/SHAM mice (Figure 2C; $\mathrm{F}_{3,20}=6.42, P=$ $0.003)$. In the transplanted VAT, IL-1 $\beta$ concentrations were significantly higher in transplants from TRANS $_{\mathrm{WT}}$ mice relative to concentrations in those from TRANS $_{\mathrm{KO}}$ mice (Figure $2 \mathrm{C} ; \mathrm{t}_{10}=7.34$, $P<0.001)$. Dietary obesity increased IL-1 $\beta$ concentrations in resi- dent VAT, but these increases were not recapitulated by VAT transplantation (Figure 2C). Quantitative PCR (qPCR) analysis of $I l 1 b$ mRNA in resident and transplanted VAT revealed similar trends, with increased expression in resident VAT from HFD/SHAM mice and in transplanted VAT from TRANS ${ }_{\mathrm{WT}}$ mice (Supplemental Figure 2C). Hippocampal Illb induction was only observed following VAT transplantation, as mice that received subcutaneous adipose tissue (SAT) transplants from a WT/HFD donor did not show changes in $I l 1 b$ mRNA levels (Supplemental Figure 2D). Il1b gene expression in SAT transplants did not differ from resident SAT in sham-operated mice (Supplemental Figure 2E), consistent with the relative immunoquiescence reported in SAT compared with VAT (16). Collectively, these data suggest that visceral adipose NLRP3 induction is required for increases in hippocampal IL-1 $\beta$ after VAT transplantation.

Visceral adipose NLRP3 induction impairs hippocampal function. For analysis of NLRP3-mediated hippocampal dysfunction after VAT transplantation, groups of LFD-fed mice received transplants from WT or NLRP3-KO donors with dietary obesity (Figure 2A). Two weeks after surgery, transplant recipients and sham-operated control mice were tested on water maze, Y-maze, and novel object recognition tasks. In the water maze, HFD/SHAM and TRANS mice had longer path lengths during acquisition training and spent less time in the target quadrant during the probe trial (Figure 2D; $\left.\mathrm{F}_{3,38}=4.68, P=0.002\right)$. The effects of VAT transplantation were

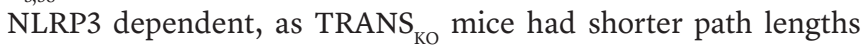
than did TRANS ${ }_{\mathrm{WT}}$ mice, and their performance did not differ from that of LFD/SHAM mice (Figure 2D). Changes in performance were not attributable to deficits in visuomotor navigation, as there were no differences for the mice when swimming toward a visible platform (distance $[\mathrm{m}]$, mean $\pm \mathrm{SEM}$ : $\mathrm{LFD} / \mathrm{SHAM}=7.29$ $\pm 0.74 ; \mathrm{HFD} / \mathrm{SHAM}=6.97 \pm 0.98 ; \mathrm{LFD} \mathrm{TRANS}_{\mathrm{WT}}=6.85 \pm 0.45$; $\left.\mathrm{LFD} \mathrm{TRANS}_{\mathrm{KO}}=6.47 \pm 0.99\right)$. We observed similar patterns in the Y-maze, where HFD/SHAM and TRANS ${ }_{\mathrm{WT}}$ mice alternated less frequently than did LFD/SHAM mice (Figure $2 \mathrm{E} ; \mathrm{F}_{3,38}=6.59, P=$ 0.001). Alternation deficits were not observed in TRANS ${ }_{\mathrm{KO}}$ mice, which did not differ from LFD/SHAM mice in this regard (Figure $2 \mathrm{E})$. In the object recognition test, HFD/SHAM and TRANS mice showed comparable reductions in novel object preference 30 minutes after training with 2 identical objects (Figure $2 \mathrm{~F} ; \mathrm{F}_{3,44}=$ $6.46, P=0.01$ ). Although within-subject reductions in novel object recognition over time were evident in all groups, TRANS ${ }_{\mathrm{KO}}$ mice spent more time exploring the novel object than did TRANS ${ }_{\mathrm{WT}}$ mice and performed similarly to LFD/SHAM mice (Figure 2F). Given that there were no differences in total object exploration (time spent with both objects [s], mean \pm SEM: LFD/SHAM = $90.88 \pm 3.97 ; \mathrm{HFD} / \mathrm{SHAM}=81.50 \pm 4.71 ; \mathrm{LFD} \mathrm{TRANS}_{\mathrm{WT}}=90.28$ $\pm 3.94 ; \mathrm{LFD}$ TRANS $_{\mathrm{Ко}}=85.03 \pm 4.99$ ), the collective outcome of these experiments is consistent with a requirement for NLRP3 in VAT transplantation-induced memory deficits.

After behavioral testing, mice were euthanized for slice preparation and extracellular recording of dentate gyrus LTP. Slice preparations from HFD/SHAM and TRANS ${ }_{\mathrm{WT}}$ mice had smaller increases in the field excitatory postsynaptic potential (fEPSP) 1 hour after tetanic stimulation (Figure $2 \mathrm{G} ; \mathrm{F}_{3,41}=6.41, P=0.001$ ). By contrast, we found that LTP in TRANS ${ }_{\mathrm{KO}}$ slices was significantly greater than in TRANS $_{\mathrm{WT}}$ slices and did not differ from 

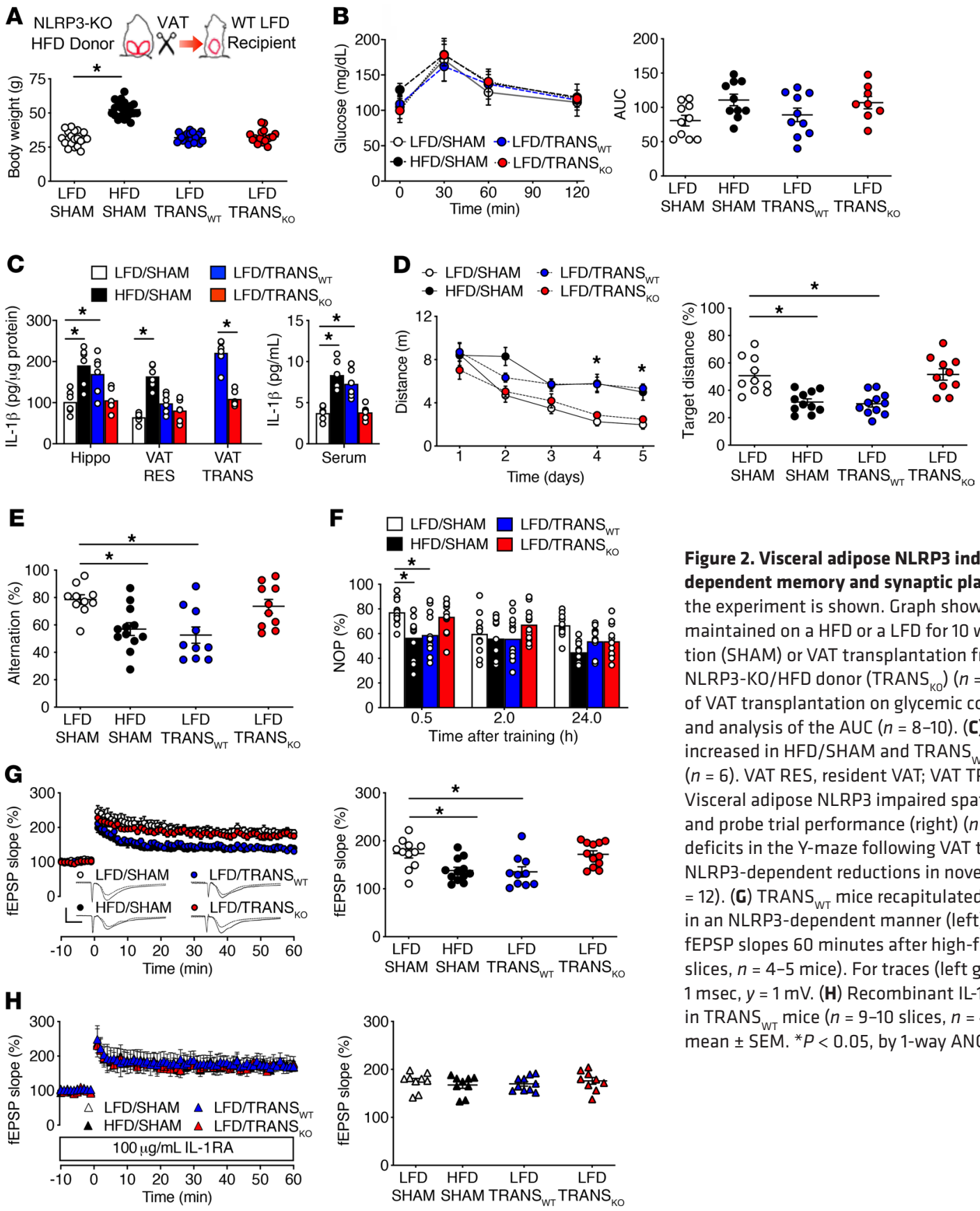

Figure 2. Visceral adipose NLRP3 induction impairs hippocampusdependent memory and synaptic plasticity. (A) Schematic of the experiment is shown. Graph shows body weights in WT mice maintained on a HFD or a LFD for 10 weeks before sham operation (SHAM) or VAT transplantation from a WT/HFD $\left(\right.$ TRANS $_{\text {WT }}$ ) or NLRP3-KO/HFD donor $\left(\right.$ TRANS $\left._{\mathrm{KO}}\right)(n=20)$. (B) There was no effect of VAT transplantation on glycemic control, as determined by IPGTT and analysis of the AUC $(n=8-10)$. (C) Hippocampal and serum IL-1 $\beta$ increased in HFD/SHAM and TRANS ${ }_{W T}$ mice, but not TRANS ${ }_{K 0}$ mice $(n=6)$. VAT RES, resident VAT; VAT TRANS, transplanted VAT. (D) Visceral adipose NLRP3 impaired spatial memory acquisition (left) and probe trial performance (right) $(n=10-11)$. (E) NLRP3-mediated deficits in the Y-maze following VAT transplantation $(n=10-12)$. ( $F)$ NLRP3-dependent reductions in novel object preference (NOP) ( $n$

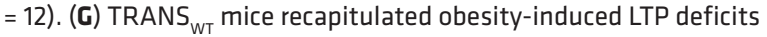
in an NLRP3-dependent manner (left), based on a comparison of fEPSP slopes 60 minutes after high-frequency stimulation ( $n=9-10$ slices, $n=4-5$ mice). For traces (left graph, inset), scale bars: $x=$ $1 \mathrm{msec}, y=1 \mathrm{mV}$. (H) Recombinant IL-1RA eliminated LTP deficits in TRANS ${ }_{W T}$ mice ( $n=9-10$ slices, $n=4-5$ mice). Data indicate the mean \pm SEM. ${ }^{*} P<0.05$, by 1 -way ANOVA with Tukey's HSD post hoc.

LTP in LFD/SHAM slices (Figure 2G). We observed no effects of diet or VAT transplantation on presynaptic paired-pulse plasticity or on the input/output ratio across a range of stimulation intensities (data not shown). To examine the role of hippocampal IL-1 in LTP deficits, we made additional recordings in the presence of recombinant IL-1 receptor antagonist (IL-1RA) $(100 \mu \mathrm{g} / \mathrm{mL})$. Preincubation with IL-1RA eliminated LTP deficits in HFD/SHAM and TRANS $_{\mathrm{WT}}$ mice without influencing LTP in LFD/SHAM or TRANS $_{\mathrm{KO}}$ mice (Figure 2H). Taken together, these results indicate that NLRP3 induction in VAT impairs cognition and suppresses LTP in an IL-1-dependent manner.

Visceral adipose NLRP3 increases penetration of peripheral $I L-1 \beta$ across the $B B B$. Coincident elevation of IL- $1 \beta$ in VAT, serum, and hippocampus could reflect changes in BBB permeability, increased transport of peripheral IL- $1 \beta$ into the CNS, or a signaling mechanism transduced by cerebrovascular cell populations. To determine whether dietary obesity and VAT transplantation regulate CNS exposure to peripheral IL-1 $\beta$, mice were i.v. injected with $10 \mu$ g 6xhistidine-tagged IL-1 $\beta$ (6xHis-IL-1 $\beta$ ) and $10 \mathrm{mg} / \mathrm{kg}$ (i.p.) of the fluorescence tracer sodium fluorescein (NaFl) (Supplemental Figure 3A). Hippocampal lysates from HFD/SHAM and TRANS ${ }_{\mathrm{WT}}$ mice exhibited greater penetration of $6 \mathrm{xHis}-\mathrm{IL}-1 \beta$ relative to LFD/SHAM mice $\left(\mathrm{F}_{3,12}=9.96, P=0.005\right.$; Supplemental Figure 3B). Increases in $6 x$ His-IL- $1 \beta$ were NLRP3 dependent, as lysates from TRANS ${ }_{\mathrm{KO}}$ mice did not differ from LFD/SHAM lysates (Supplemental Figure 3B). Changes in CNS penetration 
A

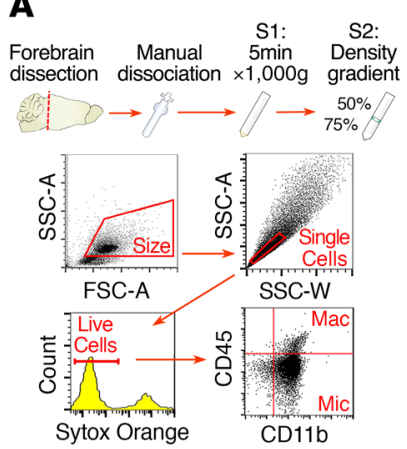

D

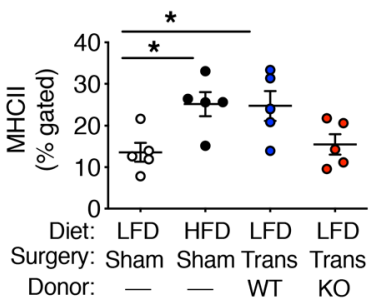

B





Surgery: Sham Sham Trans Trans

Donor: - - WT KO
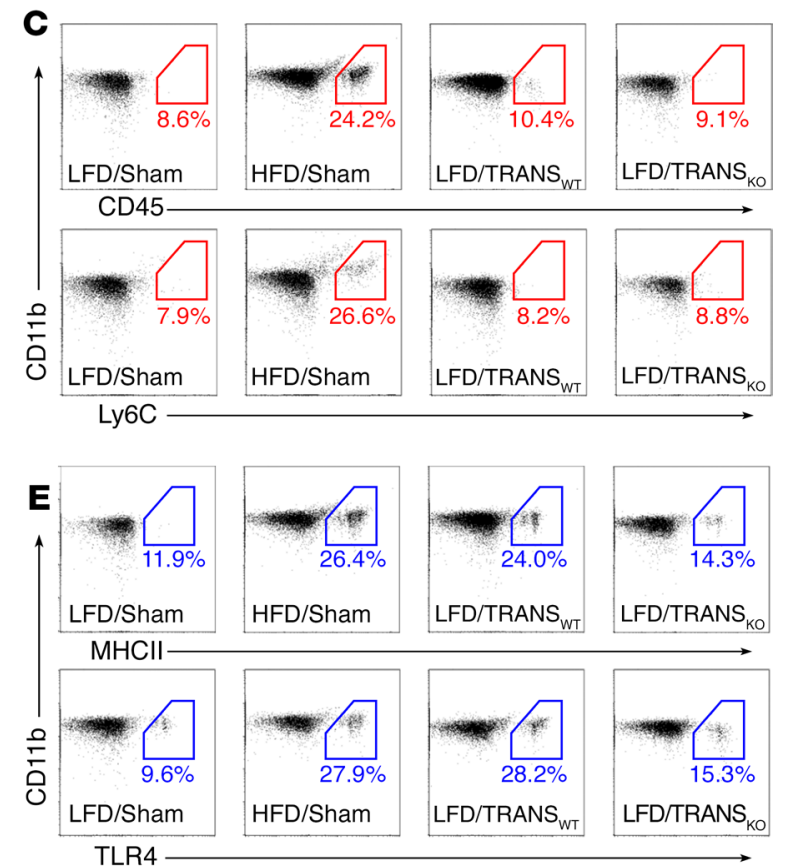
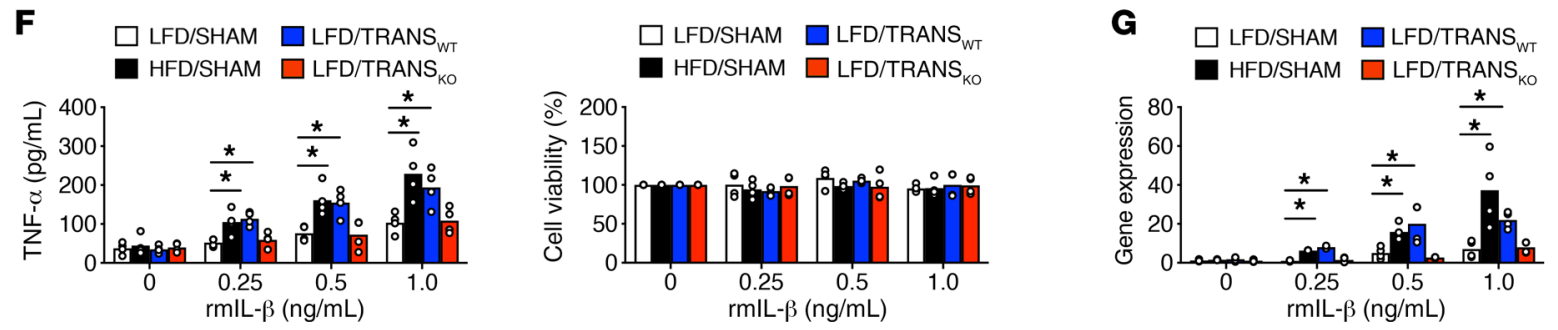

Figure 3. NLRP3-mediated effects of visceral fat transplantation on forebrain microglia. (A) Schematic of cell isolation and gating. SSC-A, side scatter area; SSC-W, side scatter pulse width; FSC-A, forward scatter area. (B) Visceral fat transplantation (Trans) did not recapitulate obesity-induced macrophage infiltration $(n=5)$. (C) Representative scatterplots of $C D 11 b^{+} C D 45^{\text {hi }}$ cells and CD11b $b^{+}$Ly6C hi cells. Boxed areas (red) show gated events. (D) VAT transplantation recapitulated the effect of obesity on microglial induction of MHCII and TLR4 via NLRP3 $(n=5)$. (E) Representative scatterplots of MHCII and TLR4 in CD11 b+ cells. Boxed area (blue) shows gated events. For all scatterplots (C and E) $x-y$ axes (log scale) minimum $=10^{\circ}$, maximum $=10^{5}$. (F) Visceral adipose NLRP3 increased sensitivity to exogenous IL-1 $\beta$ in FMCs without influencing cell viability $(n=4)$. (G) NLRP3-mediated priming of IL-1 $\beta$-stimulated gene expression with dietary obesity or VAT transplantation $(n=4)$. Data indicate the mean \pm SEM. ${ }^{*} P<0.05$, by 1 -way ANOVA with Tukey's HSD post hoc

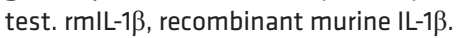

of exogenous IL-1 $\beta$ were region specific, as no group differences were observed in cortical, hypothalamic, or cerebellar lysates (Supplemental Figure 3, C-E). These patterns are consistent with increased hippocampal exposure to peripheral IL-1 $\beta$, but could have arisen as a result of $\mathrm{BBB}$ breakdown or changes in transport. Obesity increases BBB permeability $(27,28)$, and quantification of $\mathrm{NaFl}$ in hippocampal lysates from HFD/SHAM mice replicated this observation (Supplemental Figure 3F; $F_{3,12}=10.94, P<0.001$ ). However, increases in $\mathrm{BBB}$ permeability were not recapitulated by VAT transplantation (Supplemental Figure 3F), suggesting that CNS exposure to peripheral IL- $1 \beta$ in transplant recipients may have resulted from changes in transport. Collectively, these data indicate that dietary obesity and VAT transplantation promote CNS exposure to peripheral IL-1 $\beta$ via distinct mechanisms.

Cell type-specific responses to obesity and VAT transplantation. Neuroinflammation encompasses interactions between neurons, microglia, astroglia, and cerebrovascular cell populations. To gain insight into the cell type-specific effects of obesity and VAT transplantation, we isolated forebrain mononuclear cells (FMCs), astrocytes, and brain vascular endothelial cells (BVECs) for flow cytometric and gene expression analyses. Because macrophage infiltration has been reported in both genetic and dietary obesity $(29,30)$, we initially conducted immunophenotyping experiments in FMCs to determine their identity (Figure 3A). In FMCs from HFD/SHAM mice, we detected significant increases in the proportion of $\mathrm{CD} 45^{\text {hi }} \mathrm{Ly} 6 \mathrm{C}^{\mathrm{hi}} \mathrm{CD} 11 \mathrm{~b}^{+}$cells, suggestive of macrophage infiltration (Figure 3, B and $\mathrm{C} ; \mathrm{F}_{3,16}=7.75, P=0.001$ ). However, this increase was not present in FMCs from VAT transplant recipient mice (Figure 3, B and C). Following our observation that obesity, but not VAT transplantation, promotes macrophage infiltration into the brain parenchyma, we examined markers of classical activation in $\mathrm{CD}^{2} 5^{\mathrm{lo}} \mathrm{Ly} 6 \mathrm{C}^{\mathrm{lo}} \mathrm{CD} 11 \mathrm{~b}^{+}$microglia. Analysis of MHCII and TLR4 revealed evidence of microglial polarization in HFD/SHAM and TRANS ${ }_{\mathrm{WT}}$ samples (Figure 3, D and E; for MHCII, $\mathrm{F}_{3,16}=4.94$, $P=0.02$; for TLR4, $\mathrm{F}_{3,20}=5.95, P=0.02$ ). Similar to LFD $/ \mathrm{SHAM}$ mice, we observed no microglial induction of MHCII or TLR4 in 

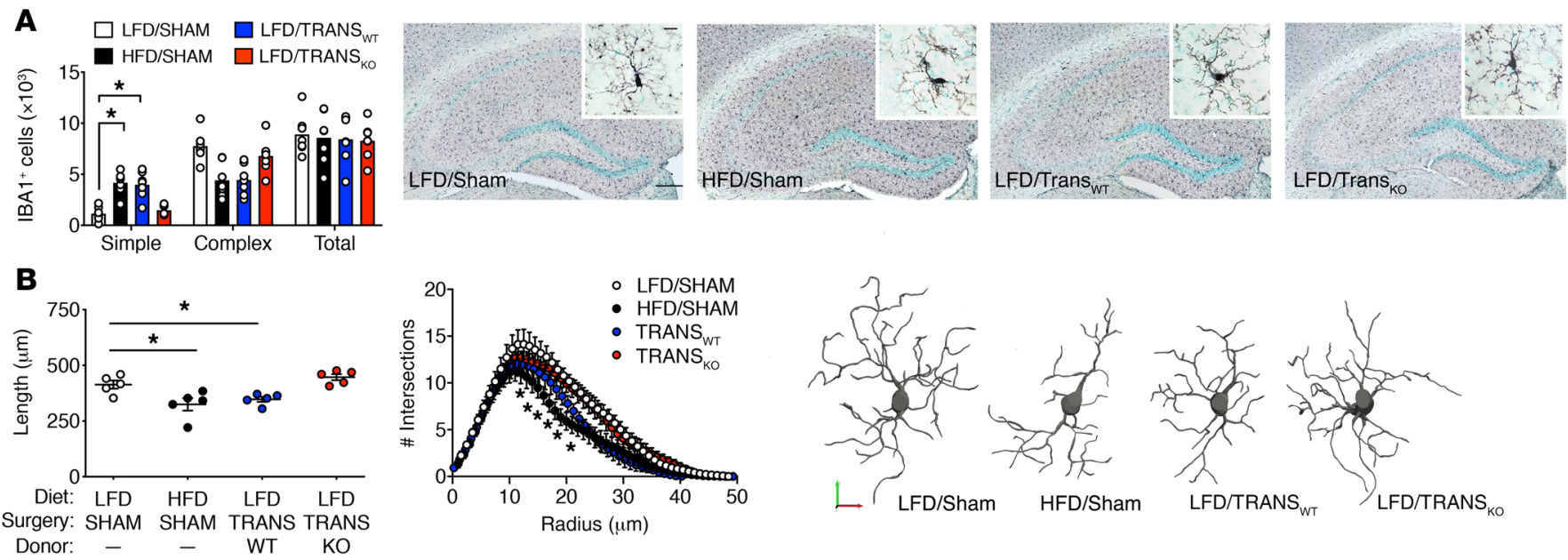

C
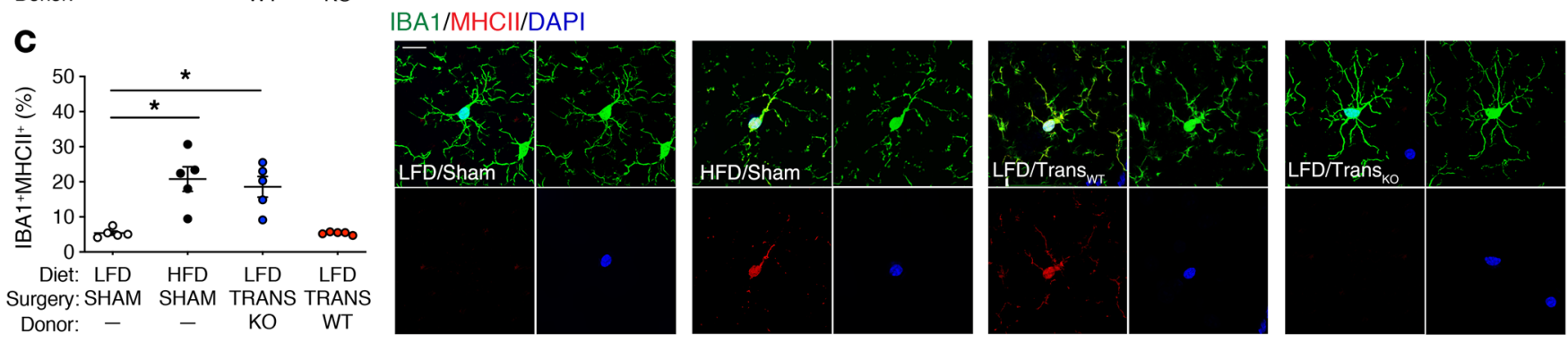

Figure 4. Visceral adipose NLRP3 induction activates hippocampal microglia in the intact brain. (A) Stereological quantification of IBA1+ microglia with 2 or fewer primary processes (Simple), more than 2 primary processes (Complex), and total number of microglia $(n=7)$. Micrographs show peroxidase detection of IBA1. Scale bars: $5 \mathrm{~mm}$ and $10 \mu \mathrm{m}$ (insets). (B) Quantification of total process length and complexity revealed NLRP3-dependent simplification of IBA1 ${ }^{+}$microglia after VAT transplantation $(n=5)$. Scale bars: $x-y-z=5 \mu \mathrm{m}$. (C) VAT transplantation recapitulated the effects of obesity on microglial MHCII induction via NLRP3 $(n=5)$. Micrographs show double-labeling for IBA1 and MHCII in the hippocampal dentate gyrus. Scale bar: $10 \mu \mathrm{m}$. Data indicate the mean \pm SEM. ${ }^{*} P<0.05$, by 1-way ANOVA with Tukey's post hoc test.

TRANS $_{\mathrm{KO}}$ mice (Figure 3, D and E), consistent with a requirement for NLRP3 in VAT transplantation-induced microglial activation.

Analysis of gene expression in FMCs, astrocytes, and BVECs revealed cell type-specific responses to obesity and VAT transplantation. We detected increases in $I l 1 b$ mRNA in FMCs and whole-hippocampal cDNA from HFD/SHAM and TRANS ${ }_{\mathrm{WT}}$ mice relative to levels in LFD/SHAM mice (Supplemental Figure 4A; for FMCs, $\mathrm{F}_{3,28}=7.75, P=0.003$; for hippocampus, $\mathrm{F}_{3,28}=5.73, P$ $=0.004)$. These effects were dependent on NLRP3, as TRANS ${ }_{\mathrm{KO}}$ samples showed reduced $I l 1 b$ levels relative to those in TRANS samples and did not differ from LFD/SHAM levels (Supplemental Figure 4A). Quantification of additional proinflammatory cytokines revealed distinct responses to HFD or VAT transplantation. Specifically, Il6 mRNA expression was increased in astrocytes and FMCs from HFD/SHAM mice relative to expression in LFD/ SHAM mice (Supplemental Figure 4B; for FMCs, $\mathrm{F}_{3,28}=5.57, P=$ 0.003; for astrocytes, $\left.\mathrm{F}_{3,28}=3.65, P=0.03\right)$. Obesity also upregulated $M c p 1$ expression in all cell types and in whole-hippocampal cDNA (Supplemental Figure 4C; for FMCs, $\mathrm{F}_{3,28}=3.51, P=0.03$; for astrocytes, $\mathrm{F}_{3,32}=3.20, P=0.04$; for BVECs, $\mathrm{F}_{3,28}=4.31, P=0.01$; for hippocampus, $\mathrm{F}_{3,28}=5.02, P=0.006$ ).

Transcriptomic studies of microglia and other CNS cell populations have reported low but detectable expression of Il1r1 under normal physiological conditions $(31,32)$. Upregulation of microglial Il1r1 has also been reported in disease models and after che- mogenetic depletion $(33,34)$. We examined cellular patterns of Il1r1 expression and immunoreactivity using immunofluorescence and qPCR (Supplemental Figure 4, D-F). Paraformaldehyde-fixed microglia, astrocytes, and BVECs from undisturbed adult WT mice were immunoreactive for IL-1R1 and phenotype-specific antigens (Supplemental Figure 4D). Quantification of Il1r1 mRNA revealed significant increases in FMCs from HFD/SHAM and TRANS mice (Supplemental Figure 4, $\mathrm{E}$ and $\mathrm{F} ; \mathrm{F}_{3,28}=9.77, P=0.001$ ). Taken together, these results implicate microglia as early detectors and potential amplifiers of visceral adipose-derived IL-1 $\beta$.

Obesity and VAT transplantation amplify microglial responses to $I L-1 \beta$. Peripheral macrophages and resident microglia show sensitization in response to proinflammatory stimuli, including IL-1 $\beta$, which primes cells for autocrine amplification (33, 35; reviewed in ref. 23). To examine whether microglia might amplify CNS responses to exogenous IL- $1 \beta$ in obesity, we measured media cytokines and gene expression in FMCs from LFD/SHAM, HFD/ SHAM, TRANS $_{\mathrm{WT}}$, and TRANS ${ }_{\text {ко }}$ mice. Cells from HFD/SHAM and TRANS ${ }_{\mathrm{WT}}$ mice had higher levels of media TNF- $\alpha$ after stimulation with increasing concentrations of IL-1 $\beta$ relative to levels in cells from LFD/SHAM mice (Figure $3 \mathrm{~F} ; \mathrm{F}_{3,48}=2.98, P=0.006$ ), indicative of sensitization. Visceral adipose NLRP3 was required for sensitization, as cells from TRANS $_{\mathrm{KO}}$ mice did not differ from LFD/SHAM mouse cells (Figure 3F). Sensitization of responses to IL- $1 \beta$ was not explained by differences in viability, as there were 
no differences in cell survival after stimulation (Figure 3F). Priming of IL-1 $\beta$-stimulated gene expression was also evident based on lower thresholds for induction of Il1 $b$ mRNA in FMCs from HFD/ SHAM and TRANS ${ }_{\mathrm{WT}}$ mice (Figure $3 \mathrm{G} ; \mathrm{F}_{3,48}=9.18, P<0.001$ ). By contrast, cells from TRANS ${ }_{\text {Kо }}$ mice exhibited IL- $1 \beta$-stimulated gene expression profiles that were comparable to those of LFD/ SHAM mice (Figure $3 \mathrm{G}$ ). When interpreted with the flow cytometric data set (Figure $3 \mathrm{~B}$ ), these outcomes suggest that the mixed population of microglia and infiltrating macrophages in FMCs from HFD/SHAM mice and the relatively homogeneous population of microglia in FMCs from TRANS $_{\mathrm{WT}}$ mice had comparable sensitization and priming in response to IL-1 $\beta$. Moreover, the lack of sensitization and priming in primary microglia from TRANS $_{\mathrm{KO}}$ mice is consistent with a requirement for NLRP3 in these effects.

Visceral adipose NLRP3 induction activates microglia in vivo. To investigate the consequences of VAT transplantation for microglia in the intact hippocampus, LFD/SHAM, HFD/SHAM, TRANS $_{\mathrm{WT}}$, and TRANS ${ }_{\mathrm{KO}}$ mice were perfused 2 weeks after surgery for immunohistochemical visualization of the microglial marker IBA1 and the activation marker MHCII. Unbiased stereological quantification of total microglial numbers in the dentate molecular layer revealed no evidence of proliferation or cell loss with dietary obesity or VAT transplantation (Figure 4A). Activated microglia typically retract their processes, and we used process number as an indicator of the activation state in these experiments. Dietary obesity and VAT transplantation were accompanied by increases in the number of "simple" microglia with 2 or fewer primary processes $\left(\mathrm{F}_{3,24}=7.14, P=0.005\right.$; Figure $\left.4 \mathrm{~A}\right)$ relative to LFD/SHAM mice. Microglial simplification was NLRP3 dependent, as counts of simple and complex ramified microglia did not differ between LFD/SHAM and TRANS ${ }_{\mathrm{KO}}$ mice (Figure $4 \mathrm{~A}$ ). 3D reconstruction of $\mathrm{IBA}^{+}$cells revealed comparable reductions in process length and complexity in TRANS ${ }_{\mathrm{WT}}$ and HFD/SHAM mice (Figure $4 \mathrm{~B}, \mathrm{~F}_{3,16}=$ 9.04, $P<0.001)$. Microglial process retraction was mediated by visceral adipose NLRP3, as TRANS ${ }_{\mathrm{KO}}$ mice had process lengths and Sholl profiles similar to those seen in LFD/SHAM mice (Figure 4B). The effects of obesity and VAT transplantation were not limited to morphology, as visualization of the activation marker MHCII in IBA1 $^{+}$cells revealed similar increases in colocalization in TRANS ${ }_{\mathrm{WT}}$ and HFD/SHAM mice (Figure 4C; $\mathrm{F}_{3,16}=12.84$, $P=0.002$ ). By contrast, TRANS $_{\text {Ко }}$ mice had significantly fewer $\mathrm{IBA}^{+} \mathrm{MHCII}^{+}$cells than did $\mathrm{TRANS}_{\mathrm{WT}}$ mice, and did not differ from LFD/SHAM mice, consistent with NLRP3-mediated microglial activation in vivo with obesity and VAT transplantation.

Generation and characterization of CX3cr $1^{\text {CreERT } I l 1 r 1^{f l / f l}-T g}$ mice. After observing correlated increases in IL-1 $\beta$, ex vivo sensitization, and microglial activation, we hypothesized that microglial IL-1R1 activation might initiate these responses in dietary obesity. To address this question, we bred $\mathrm{Tg}$ mice with inducible deletion of Il1r1 under the Cx3cr1 promoter (34). Although CX3CR1 is expressed by multiple monocyte lineages, differential turnover in resident microglia and peripheral myeloid cells enables selective manipulation of gene expression in the CNS more than 4 weeks after transgene induction (36). For additional insight into tissuespecific patterns of Il1r1 expression in this model, groups of CX3cr1 ${ }^{\text {CreERT }}$ Illr $1^{f l / f l}-\mathrm{Tg}$ mice and nontransgenic ( $\mathrm{nTg}$ ) littermates were maintained on a standard diet from weaning, with tamoxi- fen induction at 10 weeks of age and tissue collection 1 week or 4 weeks after induction (Supplemental Figure 5A). Amplification of genomic DNA from FMCs, spleen, VAT, and SAT using primers targeting the Il1r1-deleted sequence revealed excision 1 week after tamoxifen induction in all tissues (Supplemental Figure 5B). However, 4 weeks after induction, the deleted sequence was only detectable in FMCs (Supplemental Figure 5B). qPCR analysis of Il1r1 mRNA in the above tissues revealed parallel fluctuations in gene expression (Supplemental Figure 5, C-F). In FMCs from Tg mice, Il1r1 mRNA was low or undetectable after more than 40 cycles of amplification (Supplemental Figure 5C). In spleen, VAT, and SAT, transient reductions in Il1r1 mRNA were evident 1 week after induction, with complete recovery by 4 weeks (Supplemental Figure 5, D-F), indicative of selective recombination in the brain 1 month after tamoxifen administration.

Brain-specific resistance to obesity-induced inflammation in $\mathrm{CX} 3 \mathrm{cr} \mathrm{1}^{\mathrm{CrERT}} \mathrm{Il1} \mathrm{r}^{\mathrm{fl} / \mathrm{fl}}$ mice. To induce obesity, Tg mice and nTg littermates were maintained on either a HFD or a LFD for 12 weeks, with induction during week 5 (Figure $5 \mathrm{~A}$ ). Tg and $\mathrm{nTg}$ mice gained similar amounts of weight and did not show differences in glycemic control (Figure 5, A and B). Obesogenic diet consumption increased the weight of the visceral and subcutaneous fat pads, but these effects were comparable in $\mathrm{Tg}$ and $\mathrm{nTg}$ mice (Figure $5 \mathrm{C}$ ). Quantification of IL-1 $\beta$ concentrations in hippocampus, VAT, and serum revealed brain-specific resistance to IL- $1 \beta$ accumulation in Tg/HFD-fed mice (Figure 5D). nTg/HFD-fed mice exhibited increases in hippocampal IL-1 $\beta\left(F_{1 / 12}=20.83, P<0.001\right)$, but hippocampal IL-1 $\beta$ concentrations in Tg/HFD-fed mice were comparable to those in nTg/LFD-fed mice (Figure 5D). There was no effect of genotype on hippocampal IL-1 $\beta$ in LFD-fed mice, and HFD-fed mice of both genotypes had comparable increases in VAT and serum IL-1 $\beta$ (Figure 5D; for serum, $F_{1 / 12}=27.53, P<0.001$; for VAT, $\left.\mathrm{F}_{1 / 12}=23.66, P<0.001\right)$.

Increases in IL-1 $\beta$ promote transmigration of peripheral monocytes into the CNS in other disease models (37), and after observing IL-1R1-mediated increases in hippocampal IL-1 $\beta$, we sought to determine whether Tg/HFD mice might be protected against obesity-induced macrophage infiltration (Figure 5E). Analysis of cell-surface IL-1R1 expression in the $\mathrm{SSC}^{\mathrm{lo}} \mathrm{FSC}^{\mathrm{mid}}$ $\mathrm{CD} 11 \mathrm{~b}^{+} \mathrm{CD} 45^{+}$population from FMCs and lysed whole blood upheld the brain-specific recombination observed during characterization of the mouse line (Supplemental Figure 5). Both Tg/ LFD and Tg/HFD mice had comparable reductions in IL-1R1 expression relative to expression in $\mathrm{nTg}$ mice (Figure $5 \mathrm{~F}$ ). Flow cytometric immunophenotyping of FMCs revealed accumulation of CD $45^{\text {hi }}$ Ly $6 C^{\text {hi }}$ cells in $n$ Tg/HFD mice $\left(F_{1,26}=5.78, P=0.02\right)$. We did not observe increases in FMCs from Tg/HFD mice (Figure $5 G$ ), suggestive of a requirement for IL-1R1 in obesity-induced macrophage infiltration.

The CD11b ${ }^{+} \mathrm{CD} 45^{\text {hi }}$ Ly6 $\mathrm{C}^{\text {hi }}$ cell population predominantly contains infiltrating macrophages $(38,39)$, but the parent population of $\mathrm{CD} 11 \mathrm{~b}^{+} \mathrm{CD} 45^{\text {hi }}$ cells is heterogeneous in its ontogeny and origin. The meninges, choroid plexus, and perivascular space contain populations of CNS border-associated macrophages (BAMs) generated during embryonic development $(39,40)$. In the $C \times 3 \mathrm{cr} 1^{\text {CrERT }}$ line, long-lived BAMs show persistent recombination more than 4 weeks after induction (40), and to investigate responses in an 
A

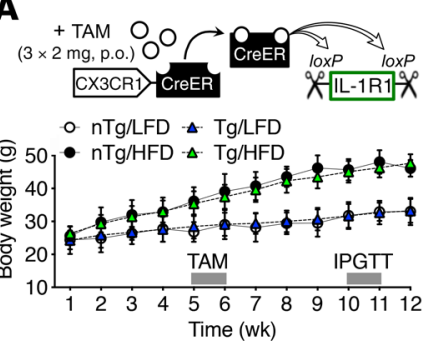

E


B



C

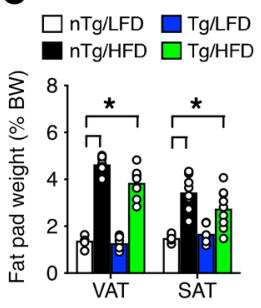

D

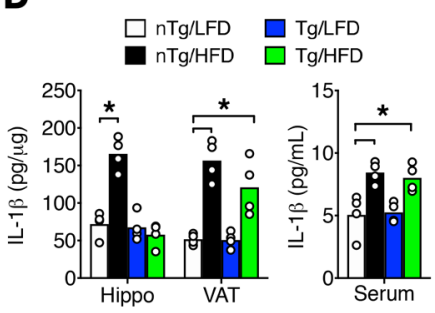

$\mathbf{H}$




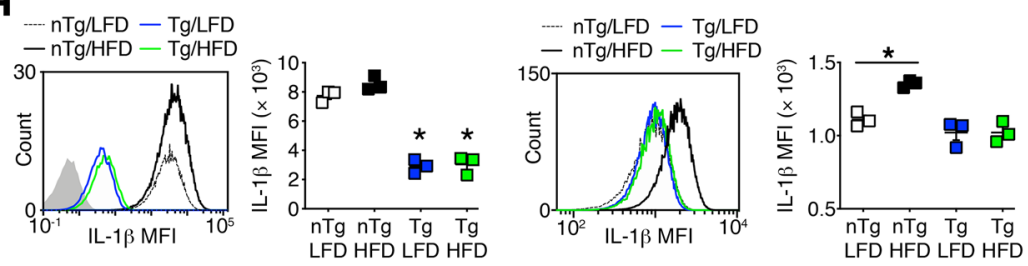

I


Figure 5. Ablation of IL-1R1 in CX3CR1-expressing cells confers resistance to obesity-induced neuroinflammation. (A) Schematic shows the design for CX3cr ${ }^{\text {TreERT2 } / / 1 r^{f l / f l}-T g}$ mice. Weight gain (graph) was similar in Tg and $n$ Tg littermates $(n=24)$. TAM, tamoxifen. (B) Genotype had no effect on glycemic control $(n=11-12)$. (C) Adipose tissue hypertrophy was unaffected by genotype $(n=6-8)$. (D) Tg/HFD mice showed no increase in hippocampal IL-1 $\beta$, despite comparable increases in VAT and serum IL-1 $\beta(n=4)$. (E) Gating strategy for analysis of macrophage infiltration and microglial activation (text above plots indicates the parent gate). (F) Flow cytometric validation of CNS-specific reductions in cell-surface IL-1R1 expression ( $n=6)$. (G) Tg mice were resistant to CNS infiltration of CD11b+CD45 hi Ly6Chi macrophages with dietary obesity $(n=7-8)$. Circles show data from cell-surface detection in live cells $(n=4-5)$, and squares represent data from fixed cells $(n=3)$. (H) Nonoverlapping effects of obesity and IL-1R1 deletion on intracellular IL-1 $\beta$ in the CD11b+ CD45 ${ }^{\text {hi }}$ Ly6C ${ }^{\text {lo }}$ cell population, which included BAMs (right; gray shading shows the isotype) and in CD11b+CD45 $5^{\circ}$ Ly6C ${ }^{10}$ microglia (left). $n=3$. (I) IL-1R1 was

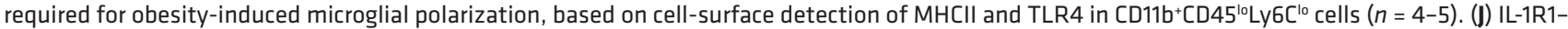
dependent anatomical simplification (left) and process retraction (right) with obesity $(n=4)$. Micrographs show IL-1R1-mediated accumulation of CD68 among IBA $1^{+}$microglia. Scale bar: $10 \mu \mathrm{m}$. Data indicate the mean \pm SEM. ${ }^{*} P<0.05$, by 2 -way ANOVA with Tukey's post hoc test.

analogous cell population, we analyzed intracellular IL-1 $\beta$ fluorescence in $\mathrm{CD}_{11} \mathrm{~b}^{+} \mathrm{CD} 45^{\text {hi }} \mathrm{Ly} 6 \mathrm{C}^{\text {lo }}$ cells. Reductions in IL-1 $\beta$ MFI were evident in $\mathrm{CD}_{11} \mathrm{~b}^{+} \mathrm{CD} 45^{\text {hi }}$ Ly6 $\mathrm{C}^{\text {lo }}$ cells from Tg mice (Figure $5 \mathrm{H} ; \mathrm{F}_{1,8}$ $=31.3, P<0.001)$. Reductions were comparable in Tg/LFD and $\mathrm{Tg} / \mathrm{HFD}$ mice, suggestive of diet-independent regulation (Figure $5 \mathrm{H})$. Quantification of IL-1 $\beta$ MFI in CD $11 \mathrm{~b}^{+} \mathrm{CD} 45^{\mathrm{lo}} \mathrm{Ly} 6 \mathrm{C}^{\mathrm{lo}}$ microglia identified nonoverlapping responses to obesity and IL-1R1 deletion. In nTg mice, we found that IL-1 $\beta$ fluorescence was weaker in microglia relative to $\mathrm{CD} 11 \mathrm{~b}^{+} \mathrm{CD} 45^{\text {hi }} \mathrm{Ly} 6 \mathrm{C}^{\text {lo }}$ cells, irrespective of diet (Figure $5 \mathrm{H}$ ). Dietary obesity increased microglial IL-1 $\beta$ MFI in nTg mice (Figure $5 \mathrm{H} ; \mathrm{F}_{1,8}=11.31, P=0.01$ ), but not in Tg mice, indicating IL-1R1-dependent mechanisms.

To determine whether differential regulation of IL-1 $\beta$ might reflect differences in activation, we quantified cell-surface MHCII


CD $45^{\text {hi }}$ Ly6C $^{\text {lo }}$ cell population, which includes BAMs (38-40). In microglia, we found that IL-1R1 was required for obesity-induced inflammatory polarization (Figure 5I; for MHCII, $\mathrm{F}_{1,14}=17.83, P=$ 0.008; for TLR4, $\left.\mathrm{F}_{1,14}=15.18, P<0.001\right)$. Among CD $45^{\text {hi }}$ Ly6C ${ }^{\text {lo }}$ cells, IL-1R1 deletion reduced MHCII and TLR4 expression in a dietindependent manner (Supplemental Figure 6, A and B; for MHCII, $\mathrm{F}_{1,14}=5.67, P=0.03$; for TLR4, $\left.\mathrm{F}_{1,14}=11.28, P=0.005\right)$. Basal expression of MHCII and TLR4 was also higher among CD45 hy6C ${ }^{\text {lo }}$ cells relative to expression in microglia (Figure 5I and Supplemental Figure 6, A and B). Although these data do not capture the potential for dynamic interactions between the 2 cell populations over time, the outcome is suggestive of distinct responses to dietary obesity and IL-1R1 deletion in microglia and brain macrophages.

We next examined IBA1 $^{+}$cells in the hippocampal dentate gyrus to determine the role of IL-1R1 in obesity-induced microglial reactivity under intact conditions. Given the morphology and location in the brain parenchyma, the IBA $1^{+}$cells sampled in these 
A $8 w k$

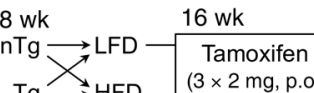

$\mathrm{Tg} \longrightarrow$ HFD $(3 \times 2 \mathrm{mg}$, p.o. $)$

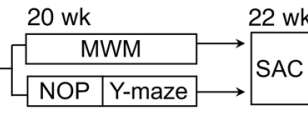

- $n T g / L F D-T g / L F D$


G

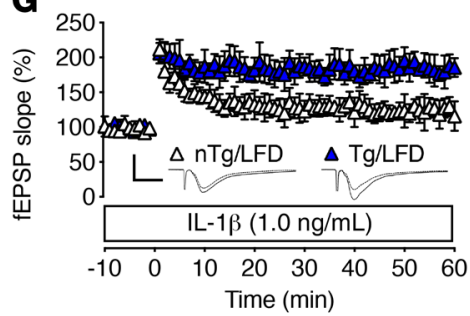



E

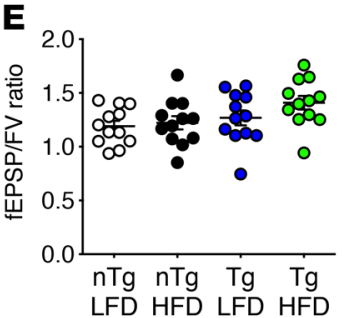

B

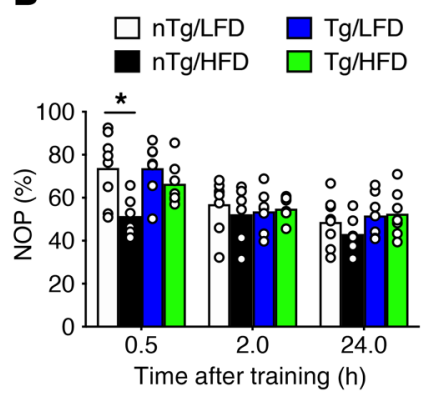

C

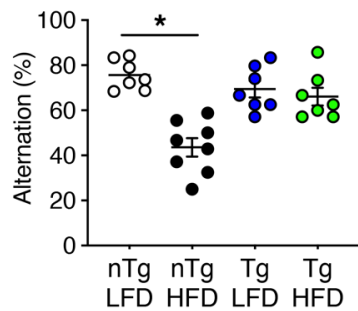


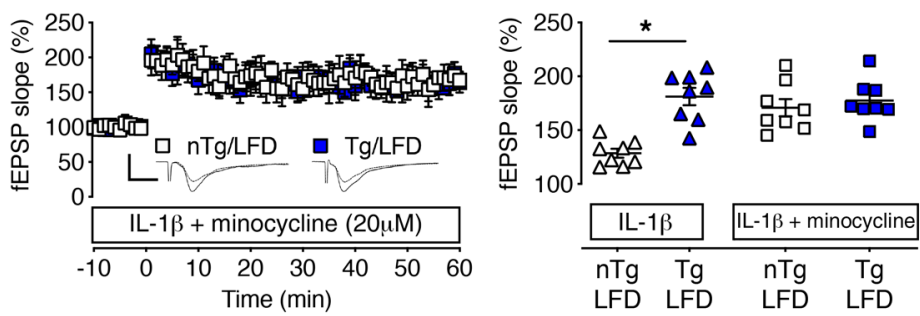

Figure 6. Protection against obesity-induced hippocampal dysfunction in CX3cr1 ${ }^{\text {CreERT }} \| / 1 r^{f l / f 1}$ mice. (A) Schematic of experiment (ages of mice are indicated). Graphs show distance during water maze acquisition (left) and probe test (right). $n=12$. MWM, Morris water maze; SAC, sacrifice. (B) IL-1R1 activation in CX3CR1-expressing cells impaired object recognition in obesity $(n=6-8)$. (C) Ablation of IL-1R1 in CX3CR1-expressing cells eliminated deficits in the Y-maze with dietary obesity $(n=7-8)$. (D) Activation of IL-1R1 in CX3CR1-expressing cells was found to underlie obesity-induced LTP deficits. $n=12$ slices, $n=5-6$ mice (D-F). For traces (insets, left graph), scale bars: $x=1 \mathrm{msec}, y=1 \mathrm{mV}$. (E) No effect of diet or genotype on input/output ratios was observed. FV, fiber volley. (F) No effect of diet or genotype on paired-pulse depression was observed. S2/S1, stimulus 2/stimulus 1. (C) Inducible ablation of IL-1R1 in CX3CR1-expressing cells protected against IL-1 $\beta$-induced LTP deficits (left graph). Preincubation with minocycline blocked reductions in LTP with exposure to IL-1 $\beta$ in slices from nTg mice (middle graph). Right graph shows normalized fEPSP slopes 60 minutes after high-frequency stimulation. For traces (insets, left and middle graphs), scale bars: $x=1 \mathrm{msec}, y=1 \mathrm{mV}$. $n=8$ slices from 4 mice per condition. Data indicate the mean $\pm S E M$. ${ }^{*} P<0.05$, by 2 -way ANOVA with Tukey's HSD post hoc test. NOP, novel object preference.

experiments likely represent resident microglia. Total process length and complexity were significantly reduced in nTg/HFD mice, but cells from $\mathrm{Tg} / \mathrm{HFD}$ mice were indistinguishable from those from $\mathrm{nTg} / \mathrm{LFD}$ control mice (Figure 5J; $\mathrm{F}_{1 / 12}=5.56, P=0.03$ ). Inducible ablation of IL-1R1 also eliminated microglial accumulation of $\mathrm{CD} 68^{+}$puncta with dietary obesity (Figure 5J). Microglia from $\mathrm{Tg} / \mathrm{LFD}$ and $\mathrm{nTg} / \mathrm{LFD}$ mice exhibited comparable morphology and CD68 immunoreactivity (Figure 5J), consistent with a requirement for IL-1R1 in obesity-induced microglial activation.

Activation of IL-1R1 on CX3CR1-expressing cells underlies hippocampal dysfunction in obesity. Following our observation that IL-1R1 was required for microglial IL- $1 \beta$ activation, we examined the consequences of this cascade for hippocampal function. Tg mice and their nTg littermates were maintained on a HFD or a LFD (Figure $5 \mathrm{~A}$ ), with behavioral testing conducted during weeks 10 through 12. In the water maze, $\mathrm{Tg} / \mathrm{HFD}$ mice were resistant to obesityinduced learning deficits, based on shorter path lengths relative to $\mathrm{nTg} / \mathrm{HFD}$ mice and intact performance during the probe trial (Figure 6A; for acquisition, $\mathrm{F}_{1,44}=7.22, P=0.01$; for probe, $\mathrm{F}_{1,44}=$
12.62, $P<0.001)$. We observed no differences between nTg/LFD and $\mathrm{Tg} / \mathrm{LFD}$ mice, and all groups performed comparably in the visible platform test (distance $[\mathrm{m}]$, mean \pm SEM: $\mathrm{nTg} / \mathrm{LFD}=5.87$ $\pm 0.4 ; \mathrm{nTg} / \mathrm{HFD}=5.62 \pm 0.7 ; \mathrm{Tg} / \mathrm{LFD}=6.20 \pm 0.5 ; \mathrm{Tg} / \mathrm{HFD}=5.2$ \pm 0.6 ). We observed similar patterns in the novel object preference paradigm, in which Tg/HFD mice were protected against deficits in recognition memory 30 minutes after training (Figure 6B; $F_{1,24}$ $=5.54, P=0.03)$. Differences in novel object preference were not explained by changes in total object exploration (time with both objects [s], mean \pm SEM: $\mathrm{nTg} / \mathrm{LFD}=83.5 \pm 5.4 ; \mathrm{nTg} / \mathrm{HFD}=76.3$ $\pm 5.6 ; \mathrm{Tg} / \mathrm{LFD}=87.1 \pm 4.9 ; \mathrm{Tg} / \mathrm{HFD}=72.7 \pm 4.5$ ), and all groups of mice exhibited within-subject decrements in object recognition over time (Figure 6B). In the Y-maze, IL-1R1 expression was required for deficits in spatial recognition, on the basis of reductions in alternation behavior in $\mathrm{nTg} / \mathrm{HFD}$, but not Tg/HFD, mice (Figure 6C; $\mathrm{F}_{1,25}=5.44, P=0.005$ ). In all paradigms, Tg/LFD mice did not differ from nTg/LFD controls, suggesting that microglial IL-1R1 may be dispensable for hippocampus-dependent memory under basal conditions. 

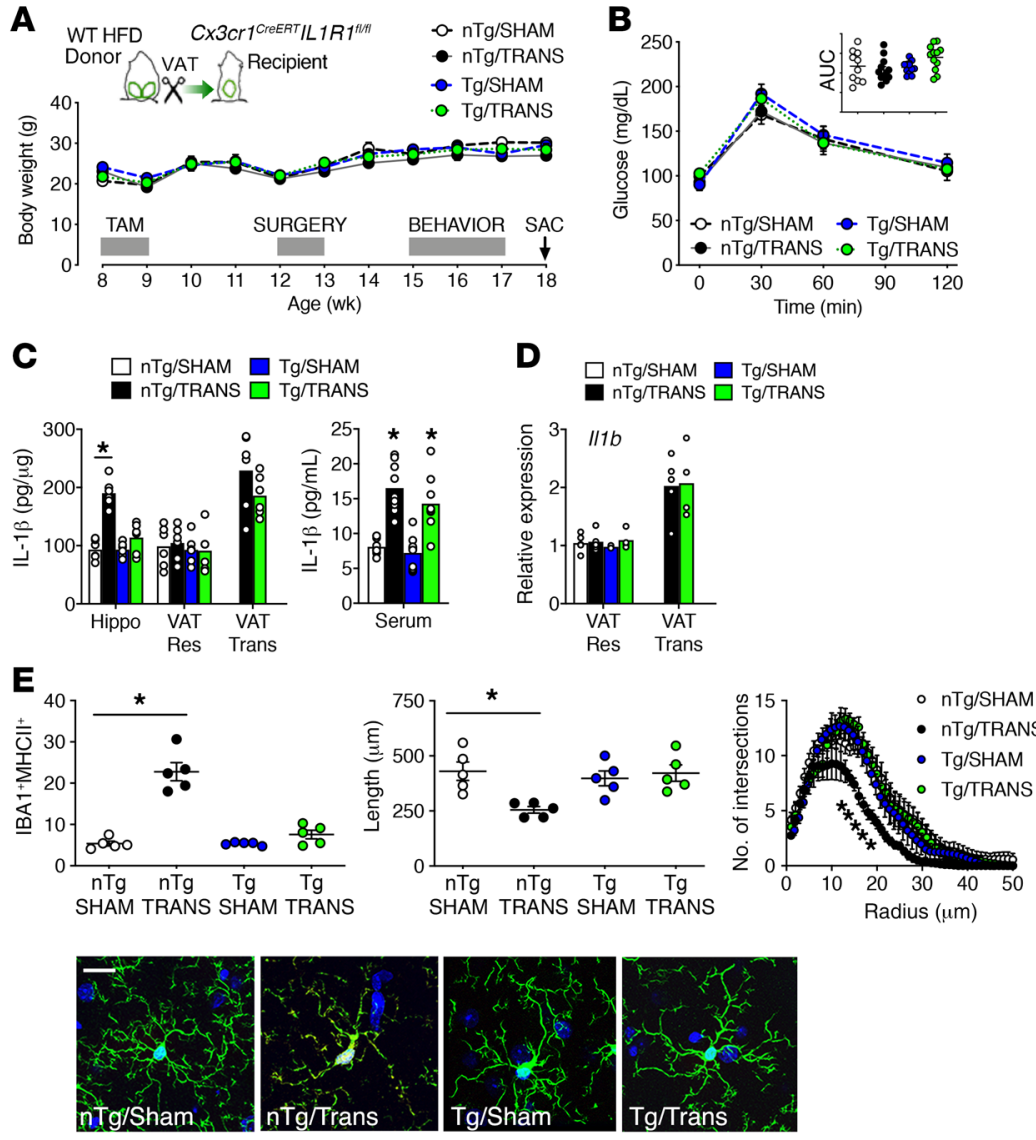

$\mathbf{F}$
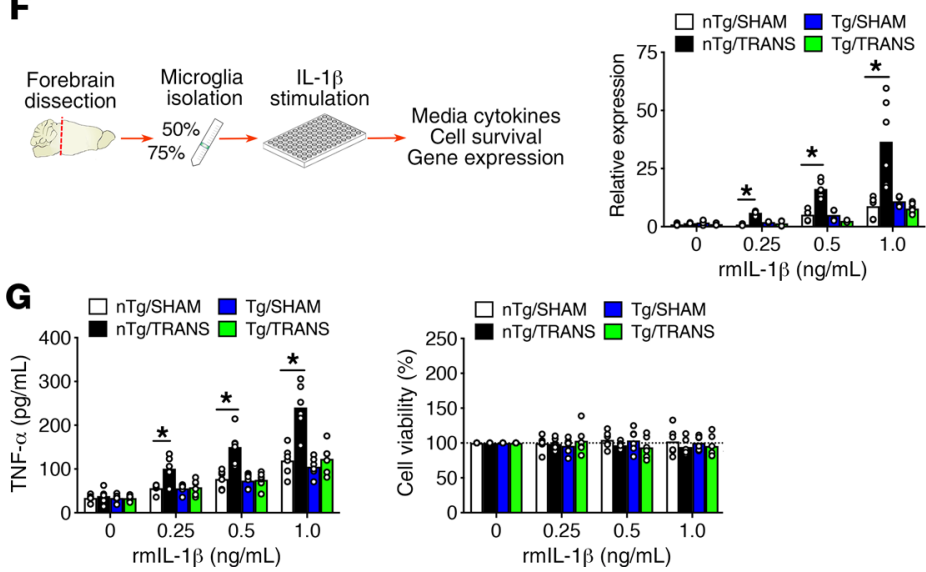

To examine the consequences of IL-1R1-mediated neuroinflammation for hippocampal synaptic plasticity, we performed extracellular field potential recordings in hippocampal slices from $\mathrm{Tg}$ mice and $\mathrm{nTg}$ littermates after HFD or LFD feeding. Induction of LTP revealed a pivotal role for IL-1R1 in obesity-induced plasticity deficits (Figure 6D).nTg/HFD mice showed significant reductions in LTP magnitude, but Tg mice on a HFD had a LTP that was comparable to that of nTg/LFD mice $\left(\mathrm{F}_{1,44}=8.86, P=\right.$ 0.008). Changes in LTP were not explained by alterations in the input/output relationship (Figure 6E), or by changes in presynaptic paired-pulse depression (Figure 6F), suggestive of postsynaptic deficits following microglial IL-1R1 activation.

Although there is consensus that immune activation-induced elevations in IL-1 $\beta$ impair LTP (41), the cellular targets for these
Figure 7. Inducible ablation of IL-1R1 in CX3CR1-expressing cells prevents microglial activation and sensitization following surgical increases in visceral fat. (A) Schematic of experiment. Graph shows weight gain after VAT transplantation (TRANS) or sham operation (SHAM) in CX3cr7 CreERT2 $/ 17 r^{f / / f l}-T g$ mice $(\mathrm{Tg})$ and $n T g$ littermates $(n=32)$. (B) Cenotype or surgery had no effect on glycemic control ( $n$ $=12$. (C) Visceral fat transplantation increased hippocampal IL-1 $\beta$ in nTg mice, but not in Tg mice, despite comparable elevations in serum IL-1 $\beta(n=6)$. (D) qPCR analysis of IL-1 $\beta$ demonstrating localized increases in transplanted VAT ( $n$ = 4-5). (E) IL-1R1-dependent MHCII induction in IBA1 ${ }^{+}$cells after VAT transplantation. IL-1R1-mediated reductions in total process length and complexity in VAT transplant recipients $(n=5)$. Micrographs show MHCIl expression in IBA $1^{+}$cells. Scale bar: $10 \mu \mathrm{m}$. (F) Schematic shows the experimental design for analysis of priming and sensitization ( $n$ = 6). Graph shows IL-1R1-mediated autocrine amplification of $1 / 1 \mathrm{~b}$ gene expression in FMCs after VAT transplantation. (C) FMCs from VAT transplant recipients exhibited IL-1R1dependent sensitization, based on lower thresholds for increases in media TNF- $\alpha$. Sensitization and priming were not attributable to differences in cell survival $(n=6)$. Data indicate the mean \pm SEM. ${ }^{*} P<0.05$, by 2 -way ANOVA with Tukey's HSD post hoc test.

effects remain poorly understood. To test the hypothesis that microglial IL-1R1 activation underlies IL-1 $\beta-$ mediated suppression of LTP, we preincubated slices from $\mathrm{nTg} / \mathrm{LFD}$ and $\mathrm{Tg} / \mathrm{LFD}$ mice with exogenous $\mathrm{IL}-1 \beta(1.0 \mathrm{ng} / \mathrm{mL})$ for 20 minutes, with continued superperfusion of IL-1 $\beta$ throughout the recording (Figure 6G). Analysis of dendritic field potentials 1 hour after tetanic stimulation revealed significant impairment of LTP in nTg/LFD slices $\left(\mathrm{t}_{14}=5.77, P\right.$ $<0.001)$. We did not observe LTP deficits in slices from $\mathrm{Tg} / \mathrm{LFD}$ mice, implicating microglial IL-1R1 activation as a mechanism for IL-1 $\beta$-mediated LTP impairment. In support of this interpretation, coapplication of the microglial inhibitor minocycline (20 $\mu \mathrm{M})$ and IL-1 $\beta$ blocked LTP deficits in slices from $\mathrm{nTg} / \mathrm{LFD}$ mice, without influencing LTP in Tg/LFD slices (Figure 6G). Taken together, these results are consistent with an obligatory role for IL-1R1 signaling among CX3CR1-expressing cells in IL-1 $\beta$-mediated synaptic dysfunction.

Direct evidence for autocrine amplification of IL-1 $1 \beta$ after VAT transplantation. To investigate the role of IL-1R1 in neuroinflammation after VAT transplantation, $\mathrm{Tg}$ mice and nTg littermates received tamoxifen 1 month before sham operation or VAT transplantation surgery (Figure 7A). Two weeks after surgery, mice were fasted for an intraperitoneal glucose tolerance test (IPGTT) before being euthanized for isolation of FMCs or immunohistological analysis of $\mathrm{IBA}^{+}$microglia. We observed no effect of genotype on weight gain after surgery (Figure 7A) and found no changes in glycemic control in Tg mice (Figure 7B). Analysis of IL-1 $\beta$ concentrations in hippocampus, serum, and VAT revealed brain-specific protection in Tg mice that received VAT transplants (Tg/TRANS). Hippocampal IL-1 $\beta$ concentrations were elevated in nTg/TRANS mice, but concentrations in Tg/TRANS mice were indistinguish- 
A

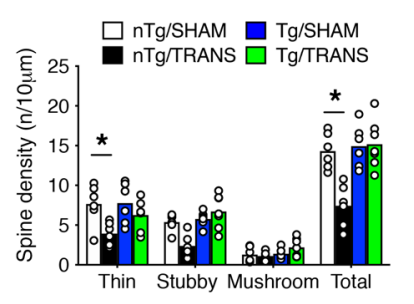

C

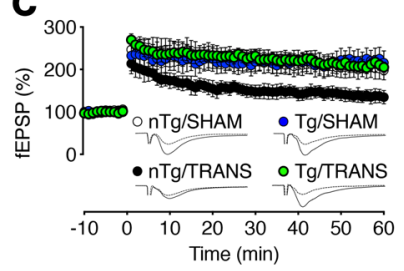

B
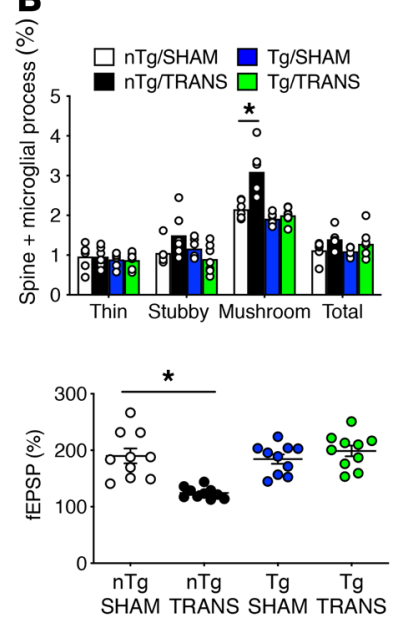

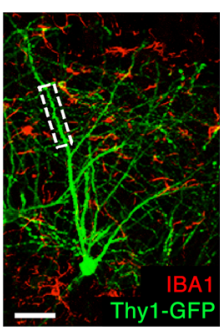

D

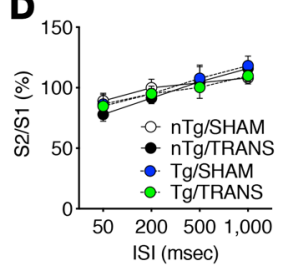



E



$\mathbf{F}$
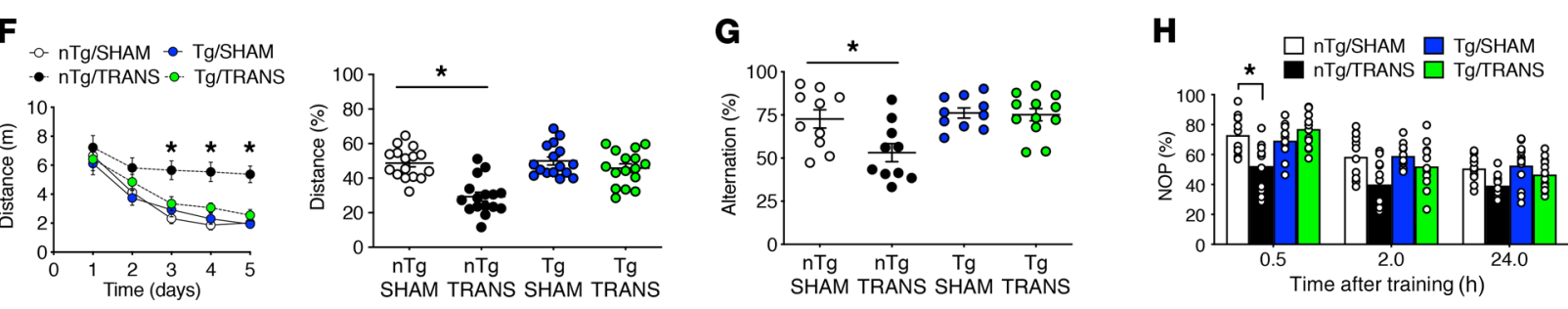

Figure 8. Visceral fat transplantation disrupts microglial organization at dendritic spines and impairs cognition by activating IL-1R1 on microglia and brain macrophages. (A) CX3cr $7^{\text {CreERT }} / / 1 r^{f / f f l}-T g$ mice were crossed with Thy-eGFP(S) mice for quantification of dendritic spine density and morphology. Loss of thin spines was associated with reductions in total dendritic spine density in nTg/TRANS mice $(n=6-7)$. (B) Analysis of IBA ${ }^{+}$microglial processes in different spine morphologies revealed preferential localization at mushroom spines. VAT transplantation increased the proportion of mushroom spines with microglial processes, and increases were IL-1R1 dependent. For micrographs, left panel shows a Z-projection of Thy1-GFP and IBA1 (scale bar: $20 \mu \mathrm{m}$ ); middle panels show individual Z-planes, with asterisks indicating contact between IBA1 ${ }^{+}$processes and dendritic spines (scale bar: $25 \mu \mathrm{m}$ ). Far right panels show representative $Z$-projection images from each condition, with arrowheads indicating spines and asterisks indicating IBA1 contact at the spine head (scale bar: $10 \mu \mathrm{m}$ ). $n=6-7$. (C) Protection against VAT transplantation-induced LTP deficits in Tg/TRANS mice (left), based on comparison of fEPSP slopes 60 minutes after high-frequency stimulation (right). (D) No change in presynaptic paired-pulse plasticity was observed. ISI, interstimulus interval. $n=10$ slices, $n=4-5$ mice. (E) No effect of genotype or surgery on input/output ratios was observed. (F) VAT transplantation impaired water maze acquisition (left) and probe trial performance (right) in nTg/TRANS, but not Tg/TRANS, mice $(n=16)$. (G and $\mathbf{H})$ Ablation of IL-1R1 in CX3CR1-expressing cells eliminated VAT transplantation-induced deficits in the $\mathrm{Y}$-maze $(\mathbf{C})$ and maintained object recognition memory $(\mathbf{H}) . n=10-12$. Data indicate the mean \pm SEM. ${ }^{*} P<0.05$, by 2-way ANOVA with Tukey's HSD post hoc.

able from those in sham-operated mice (Figure $7 \mathrm{C} ; \mathrm{F}_{1,20}=6.38, P=$ 0.02). Transplant recipients had increased serum IL-1 $\beta$ relative to sham-operated mice, but increases in circulating IL-1 $\beta$ were unaffected by genotype (Figure 7C). Concentrations of IL-1 $\beta$ were significantly higher in the transplanted VAT relative to resident VAT, but there was no effect of recipient genotype on IL-1 $\beta$ levels in either set of samples (Figure 7C). Fluctuations in adipose IL-1 $\beta$ protein concentrations were paralleled by changes in Illb gene expression, which was significantly $\left(\mathrm{F}_{1 / 20}=22.87, P=0.005\right)$ elevated in transplanted VAT, independent of recipient genotype (Figure 7D). Following our observation of protection against hippocampal IL-1 $\beta$ accumulation in Tg/TRANS mice, we analyzed microglial morphology and MHCII induction. We found that VAT transplantation increased the number of $\mathrm{IBA}^{+} \mathrm{MHCII}^{+}$cells in $\mathrm{nTg}$ mice, but not in Tg mice (Figure 7E; $\mathrm{F}_{1,16}=24.52, P<0.001$ ). Changes in MHCII immunoreactivity were accompanied by IL-1R1-mediated reductions in microglial process length and complexity (Figure 7E; $F_{1,16}$ $=8.85, P=0.008)$. Collectively, these results indicate that IL-1R1 expression in CX3CR1-expressing cells is required for neuroinflammation following surgical increases in visceral fat.
After observing an obligatory role for IL-1R1 in hippocampal IL-1 $\beta$ accumulation, we investigated the potential requirement for IL-1R1 in autocrine amplification of IL-1 $\beta$ ex vivo. Analysis of gene expression in FMCs after stimulation with increasing concentrations of IL-1 $\beta$ revealed IL-1R1-mediated priming in transplant recipients (Figure 7F). Cells from nTg/TRANS mice had lower thresholds for induction of $I l 1 b$ mRNA, but cells from $\mathrm{Tg}$ /TRANS mice were comparable to those from nTg/SHAM mice (Figure 7F; $F_{1,20}=48.28, P<0.01$ ). Priming of gene expression was accompanied by IL-1R1-mediated sensitization, based on increases in media TNF- $\alpha$ in cells from nTg/TRANS, but not $\mathrm{Tg}$ /TRANS, mice (Figure 7G; $\mathrm{F}_{1,20}=19.63, P=0.002$ ). Changes in sensitization and priming were not attributable to differential cell survival, since there were no group differences, as determined by a formazan cleavage assay (Figure 7G). The effect of genotype was only evident in transplant recipients, as cells from $\mathrm{Tg} / \mathrm{SHAM}$ mice did not differ from those of nTg/SHAM mice (Figure 7, F and G). Taken together, these patterns are consistent with IL-1R1-mediated autocrine amplification of $I l 1 b$ expression following VAT transplantation. 
Protection against obesity-induced microglial activation in $\mathrm{CX} 3 \mathrm{cr} 1^{\mathrm{CrER}} \mathrm{Il1r} 1^{\mathrm{fl} / \mathrm{fl}}$ mice. Microglia interact with neurons via receptor-mediated signaling, alone or in concert with physical interactions with synaptic terminals $(25,42,43)$. We previously reported that dietary obesity disrupts the organization of microglial processes around hippocampal synaptic terminals (26). To investigate whether VAT transplantation might recapitulate these effects and examine the potential contributions of IL-1R1, we crossed Tg mice with the Thy1-eGFP(S) line to generate $C x 3 c r 1^{\text {CreERT } I l 1 r 1^{f / f l} \text { mice }}$ with endogenous fluorescence in dentate granule neurons (Figure $8, \mathrm{~A}$ and B). Semiautomated detection and quantification of dendritic spines revealed reductions in total dendritic spine density after VAT transplantation (Figure $8 \mathrm{~A} ; \mathrm{F}_{1,21}=10.92, P=0.003$ ). These effects were primarily attributable to loss of thin spines and were mediated by IL-1R1 activation among CX3CR1-expressing cells (Figure 8, A and $\mathrm{B} ; \mathrm{F}_{1,21}=9.01, P=0.007$ ). Parallel visualization of IBA1 revealed preferential localization of microglial processes at mushroom spines relative to other morphologies in all groups of mice (Figure 8B; number of spines sampled per animal, mean $\pm \mathrm{SEM}=551 \pm 75.2$ ). This pattern was further amplified by VAT transplantation in $\mathrm{nTg} / \mathrm{TRANS}$ mice $\left(\mathrm{F}_{1,21}=10.08, P\right.$ $=0.004)$. The effects of VAT transplantation on microglial apposition at mushroom spines were IL-1R1 dependent, as Tg/TRANS mice had similar proportions of mushroom spines with perisynaptic microglial processes relative to $\mathrm{nTg} / \mathrm{SHAM}$ mice (Figure $8 \mathrm{~B}$ ). IL-1R1-mediated perturbation of microglia-synapse relationships was only evident after VAT transplantation, as Tg/SHAM mice did not differ from $\mathrm{nTg} / \mathrm{SHAM}$ in terms of spine density or microglial localization (Figure 8, A and B). Collectively, these results are consistent with a multifaceted role for IL-1R1 in regulating microglia-synapse interactions following surgical increases in visceral fat.

To examine the functional consequences of IL-1R1-mediated changes in microglia-synapse relationships, we performed extracellular recordings in brain slices and found that dentate gyrus LTP was significantly reduced in slices from nTg/TRANS mice but not in those from Tg/TRANS mice (Figure 8C; $F_{1,40}=10.56$, $P=0.003)$. Presynaptic paired-pulse plasticity was unaffected at interpulse intervals ranging from $50 \mathrm{msec}$ to 1 second (Figure 8D), and comparison of the input/output curve over a range of stimulus intensities revealed no effect of genotype or surgery (Figure 8E). Analysis of hippocampus-dependent memory in the water maze revealed that IL-1R1 was required for memory deficits after VAT transplantation (Figure 8F). Tg/TRANS mice had shorter swim paths than did nTg/TRANS mice during acquisition training and performed on par with nTg/SHAM mice during the probe trial (Figure 8F; for acquisition, $\mathrm{F}_{3,56}=9.09, P=0.003$; for probe, $\mathrm{F}_{1,56}=$ $5.55, P=0.03)$. We observed no differences between nTg/SHAM and $\mathrm{Tg} / \mathrm{SHAM}$ mice during acquisition training and no effect of genotype or VAT transplantation on navigation toward the visible platform (distance $[\mathrm{m}]$; mean $\pm \mathrm{SEM}, \mathrm{nTg} / \mathrm{SHAM}=6.78 \pm 1.25$; $\mathrm{nTg} / \mathrm{TRANS}=7.27 \pm 0.98 ; \mathrm{Tg} / \mathrm{SHAM}=6.46 \pm 1.49 ; \mathrm{Tg} / \mathrm{TRANS}=$ $7.21 \pm 0.77)$. Ablation of IL-1R1 also eliminated deficits in spontaneous alternation in the Y-maze after VAT transplantation (Figure $\left.8 G ; \mathrm{F}_{1,38}=4.55, P=0.04\right)$. There were no differences in alternation between $\mathrm{nTg} / \mathrm{SHAM}$ and $\mathrm{Tg} / \mathrm{SHAM}$ mice (Figure $8 \mathrm{G}$ ). In the object recognition task, $\mathrm{Tg}$ /TRANS mice performed significantly better than did $\mathrm{nTg} / \mathrm{TRANS}$ mice at the 30 -minute post-training time point (Figure $8 \mathrm{H} ; \mathrm{F}_{3,43}=9.52, P=0.005$ ). We found no differences in object recognition between nTg/SHAM and Tg/SHAM mice, and all groups of mice exhibited within-subject reductions in novel object preference over time (Figure $8 \mathrm{H}$ ). Taken together, these data indicate that IL-1R1 activation among CX3CR1expressing cells underlies VAT transplantation-induced deficits in hippocampus-dependent memory.

\section{Discussion}

Although there is consensus that obesity elicits proinflammatory responses in peripheral tissues, interpretation of similar changes in the brain is complicated by longstanding assumptions regarding immune privilege in the nervous system. The current studies add to a growing body of work challenging these assumptions by demonstrating that NLRP3 inflammasome activation in VAT impairs cognition in obesity. These effects were mediated by activation of IL-1R1 in CX3CR1-expressing cells, which detect and amplify IL-1 $\beta$ in the brain. Given that aging, Alzheimer's disease, and obesity are associated with NLRP3 induction and local inflammation in multiple tissues, including the CNS (20, 21, 24), it is surprising that tissue-specific requirements for NLRP3 remain poorly understood. To our knowledge, the VAT transplantation studies in this report represent the first regionally selective manipulation of NLRP3 to examine tissue-specific regulation of synaptic plasticity and cognition.

Visceral adipose NLRP3 induction promotes caspase-1-mediated cleavage of pro-IL-1 $\beta$, leading to the release of mature IL- $1 \beta$ from adipose tissue macrophages $(18,44,45)$. Multiple lines of evidence in this report suggest that exposure to peripherally generated IL- $1 \beta$ initiates local amplification by brain-resident immune cells, similar to that reported following intracerebroventricular IL-1 $\beta$ injections and in experimental autoimmune encephalomyelitis $(33,46)$. However, it is also possible that CNS BAMs in the meninges and perivascular space could instigate or oppose parenchymal responses to IL-1 $\beta$. Long-lived BAMs exhibit recombination at extended time points after induction in the CX3CR $1^{\text {CreERT }}$ line (40), and interactions between BAMs and cerebrospinal fluid (CSF) immune cells were previously shown to regulate cognition (47). In light of these patterns, it is possible that obesity-induced cognitive deficits could arise as a result of IL-1R1 activation on BAMs, alone or in concert with responses among resident microglia. In one potential scenario, IL-1R1 activation would promote synthesis and release of IL-1 $\beta$ by BAMs, which would reach the hippocampus directly by volume transmission, or indirectly via paracrine signaling interactions with adjacent cells $(46,48)$. Although the indirect hypothesis cannot be ruled out, the timeframe for changes in hippocampal synaptic plasticity following ex vivo manipulation of IL-1 signaling suggests a more proximal interaction between CX3CR1-expressing cells and synaptic terminals. Perivascular macrophages represent the most likely subpopulation of BAMs for these effects, given their parenchymal location and instigative role in neuroinflammation following peripheral infection $(49,50)$. However, in obesity, peripheral inflammation develops more slowly than in models of acute infection, and the contributions of perivascular macrophages to chronic (neuro) inflammation are less certain. The difficult questions surrounding the origins of neuroinflammation in obesity are emblematic of 
larger gaps in knowledge related to CNS immune privilege and the degree of interaction between parenchymal cells and circulating factors. Although the subpopulation of CX3CR1-expressing cells that initiates obesity-induced cognitive dysfunction remains to be determined, the current study clearly implicates IL-1R1 activation in brain-resident immune cells as a link between visceral adipose NLRP3 induction and cognitive impairment.

Within the CNS, IL-1R1 is highly expressed among vascular endothelial cells, with lower but detectable expression in microglia, astrocytes, and neurons (31-34, 51-53). Microglial IL-1R1 expression is upregulated following chemogenetic depletion and in animal models of chronic inflammation $(33,34)$, consistent with a central role for IL-1 signaling in repopulation and resolution of neuroinflammation. The downstream effects of IL-1R1 activation in the brain are determined by cell type-specific expression of splice variants for the IL-1 receptor accessory protein (IL-1RAcP). The AcP variant (IL-1RAcp) expressed by glia is identical to the coreceptor expressed in peripheral tissues, whereas the $\mathrm{AcPb}$ variant (IL-1RAcPb) is exclusively expressed by neurons $(54,55)$. Both receptors have homologous extracellular regions, but the $\mathrm{AcPb}$ variant is unable to recruit the adapter protein MyD88, which is required for downstream induction of proinflammatory signaling cascades (23). Consistent with this, exogenous IL-1 $\beta$ recruits NF- $\mathrm{BB}-$ mediated transcriptional responses in glial cultures, but not in primary neurons (52). Interestingly, aging is accompanied by neuronal induction of the proinflammatory $\mathrm{AcPb}$ variant (56), suggesting that chronic exposure to low-level inflammation might reprogram neuronal IL-1RAcP expression in other disease states. Although the potential contributions of neuronal IL-1RAcP reconfiguration remain unexplored in obesity, it is tempting to speculate that a switch in AcP splice variant expression might occur downstream of local amplification of IL-1 $\beta$ among CX3CR1-expressing cells in these experiments.

An extensive body of work indicates that exposure to the levels of IL-1 $\beta$ seen in chronic inflammatory diseases impairs hippocampal synaptic plasticity and cognition (refs. 57-59; reviewed in ref. 41). The relationship between IL-1 $\beta$ and hippocampal synaptic function is biphasic, with enhancement of synaptic plasticity following picomolar stimulation and deficits occurring after exposure to higher concentrations $(54,58,60)$. Consistent with a facilitative role for low-level IL-1R1 activation in synaptic physiology, wholebody IL-1R1-KO mice exhibit deficits in hippocampus-dependent memory and LTP (61). The dose-dependent effects of IL-1 $\beta$ on hippocampal synaptic plasticity have been interpreted as reflecting direct actions on neurons, but this assumption has yet to be adequately tested with modern cell type-specific approaches. In this report, we observed that ablation of IL-1R1 in CX3CR1expressing cells protects against IL- $1 \beta$-mediated deficits in LTP. The effects observed in normal-weight IL-1R1-Tg mice were similar to the protective effects of preincubation with minocycline, a broadspectrum tetracycline antibiotic used experimentally to inhibit microglial activation $(62,63)$. Taken together, these results suggest that the biphasic relationship between IL-1 $\beta$ and hippocampal synaptic plasticity may reflect activation of IL-1R1 on different cell types, with opposing consequences for memory and cognition.

Microglia regulate neuronal function indirectly by clearing dead cells and extracellular debris, and directly by releasing sig- naling molecules that support or suppress neuroplasticity (36). Interactions between microglia and neurons may also involve physical interposition of microglial processes between pre- and postsynaptic structures, alone or in concert with microglial internalization of synaptic terminals $(25,42)$. It is unlikely that microglial localization at mushroom spines in this study reflects synaptic stripping, given that a recent large-scale analysis of hippocampal dendritic spines using correlated light and electron microscopy revealed no evidence of postsynaptic internalization (43). VAT transplantation was associated with IL-1R1-mediated loss of thin spines in these studies, consistent with our previously published ultrastructural studies in genetically obese $\mathrm{db} / \mathrm{db}$ mice (64). However, any potential relationship between loss of thin spines and IL-1R1-mediated microglial activation remains speculative at present. Understanding and manipulating glial interactions with synapses and circuits are formidable challenges with the potential to uncover novel strategies for the prevention and treatment of neurological disease.

In humans, there is consensus that insulin resistance is accompanied by an increased risk of cognitive decline (65). By contrast, data from studies of BMI and cognition are often conflicting, with reports of increased risk (1-5), decreased risk (9), or negative findings $(10,11,66)$. The results of the current study could potentially underlie the reported associations between visceral adiposity and cognitive decline in human studies $(4,13,14)$. Given that a growing literature from longitudinal and twin studies supports a potential link between visceral adiposity and age-related cognitive decline, intervention studies may be warranted to prevent or attenuate risk. Although there is a clear positive effect of lifestyle interventions, including the Mediterranean diet and exercise $(67,68)$, long-term compliance can be difficult to achieve for most individuals. Surgical interventions such as Roux-en-Y gastric bypass, vertical banded gastroplasty, and laparoscopic adjustable gastric banding have begun to receive greater attention with respect to postoperative changes in memory and cognition. In a recent meta-analysis, significant improvements in attention, mood, and executive function were detected after bariatric surgery in morbidly obese patients (69). Cognitive improvements were independent of reductions in BMI, whereas reductions in circulating markers of inflammation were correlated with cognitive change in some, but not all, studies $(70,71)$. Bariatric surgery is sometimes performed together with surgical removal of fat from the visceral omentum (omentectomy). Although most studies did not report any metabolic improvements following omentectomy alone or with concurrent gastric bypass surgery (refs. 72, 73; reviewed in ref. 74), omentectomy has not been studied with respect to the regulation of cognition in humans. For mild to moderate obesity, surgical interventions will generate more risk than reward, even in the context of long-term vulnerability to age-related cognitive decline. However, studying brain circuits that respond to weight loss surgery could uncover novel targets for noninvasive modulation using transcranial magnetic stimulation and other emerging technologies.

\section{Methods}

Animals and diets. To induce obesity, male mice were maintained on a HFD (Research Diets, D12492) or a LFD (Research Diets, D12450) beginning at 8 weeks of age. WT and NLRP3-KO mice (Jax 
strain no. 021302) were obtained from The Jackson Laboratory or bred in-house for these experiments. Mice with inducible ablation

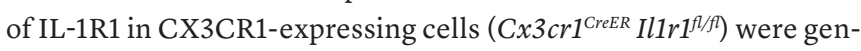
erated by crossing IL-1R1-floxed mice (provided by Ari Waisman, University of Mainz, Mainz, Germany; ref. 34) with $C x 3$ cr $1^{\text {CreER }}$ mice purchased from The Jackson Laboratory (strain no. 021160). For some experiments, Cx3cr1 ${ }^{\text {CreER }}$ Il1r $1^{f / f l-T g}$ mice were crossed with the Thy1-eGFP(S) mouse line (The Jackson Laboratory, strain no. 011070; breeding pairs were donated by Lin Mei, Augusta University, Augusta, Georgia, USA) to generate Tg mice with endogenous fluorescence in the dendritic arbor of dentate granule neurons. Tg mice and $\mathrm{nTg}$ littermates were administered tamoxifen $(2 \mathrm{mg}$ in $0.2 \mathrm{~mL}$ corn oil, p.o.) 3 times every 48 hours. Additional details on housing conditions, genotyping, and animal care are provided in the Supplemental Methods.

Fat transplantation and determination of transplant viability. For WAT transplantation, the epididymal or inguinal fat pads were removed and trimmed to $300 \mathrm{mg}$ before transplantation into the peritoneal cavity of recipient mice, as described previously (75). Transplants were collected upon euthanization for histological verification of transplant viability, as described in the Supplemental Methods.

Behavioral testing. Testing in the Y-maze and novel object preference tasks was carried out as described previously $(26,29)$. Hippocampus-dependent memory was assessed using the water maze, as reported previously $(76,77)$. Data acquisition was performed by an experimenter blinded to the experimental condition, with the exception of the visually evident phenotype in HFD-fed mice. Data analysis was performed in a blinded manner using ANY-maze behavioral tracking software (Stoelting Co.).

$B B B$ permeability and IL-1 $\beta$ transport. Mice were injected $6 \mathrm{xHis}-$ IL-1 $\beta$ (1.0 $\mu$ g, i.v.; USBiological) and the fluorescence tracer NaFl (10 $\mathrm{mg} / \mathrm{kg}$, i.p.; MilliporeSigma) before euthanization by transcardial perfusion with saline, as described previously (28). The dose and route of administration for $6 \mathrm{xHis}-\mathrm{IL}-1 \beta$ were selected on the basis of an absence of acute IL-1-mediated effects on BBB permeability (78). Detection and quantification of $\mathrm{NaFl}$ were performed according to previously published methodology (28).

ELISA and Western blotting. Protein extraction and quantification of IL- $1 \beta$ and TNF- $\alpha$ by ELISA were performed according to previously published protocols (ref. 79 and Supplemental Methods). Methods for SDS-PAGE and Western blotting were modified from previously published protocols $(28,75)$ and are described in the Supplemental Methods.

Immunofluorescence, confocal microscopy, and morphological analysis. For details regarding immunofluorescence reactions, see the Supplemental Methods. Images were acquired on a Zeiss 780 multiphoton microscope and analyzed by a blinded experimenter. Details on cell sampling and analysis are provided in the Supplemental Methods.

Cell isolation and ex vivo stimulation. Cells were isolated according to published protocols (28) before labeling with conjugated antibodies for flow cytometry or frozen for gene expression endpoints (see Supplemental Methods). The methods for ex vivo stimulation experiments were modified from published studies $(75,79)$ and are described in the Supplemental Methods.

Flow cytometry. For cell-surface markers, immunophenotyping was carried out as described previously (ref. 29 and Supplemental Methods). For intracellular flow cytometric analysis, live cells were incubated with conjugated antibodies against CD45, Ly6C, and $\mathrm{CD} 11 \mathrm{~b}$ before fixation and permeabilization using commercial reagents (Affymetrix eBioscience). Cells were then washed and incubated with primary antibodies for intracellular detection of IL-1 $\beta$ (Santa Cruz Biotechnology). Viable cells were visibly differentiated from debris by size gating and positivity for specific antibodies using a BD LSR II Flow Cytometer (BD Biosciences). Single stains were used to set compensation, and isotype controls were used to determine the level of nonspecific binding. Analysis was performed using FlowJo, version 11.0 (BD Biosciences) or InCyte (MilliporeSigma) software.

$R N A$ isolation and $q P C R$. Total RNA extraction and cDNA synthesis were performed according to published methodology (refs. 28, 64 and Supplemental Methods).

Hippocampal slice preparation and electrophysiology. Hippocampal slice preparation and extracellular recording were carried out as described (refs. 28-29, 75 and Supplemental Methods).

Statistics. For comparisons between LFD/SHAM, HFD/SHAM, TRANS $_{\mathrm{WT}}$, and TRANS $_{\text {Kо }}$ mice, data were analyzed with a 1-way ANOVA or 1-way repeated-measures ANOVA followed by Tukey's honestly significant difference (HSD) post hoc test. For repeated-measures endpoints, data were analyzed using a repeated-measures ANOVA and Tukey's HSD post hoc test. For experiments comparing the effects of a HFD or VAT transplantation in $C \times 3 \mathrm{cr} 1^{\text {CreERT2 }} \mathrm{Il1r} 1^{f / f l}-\mathrm{Tg}$ mice and $\mathrm{nTg}$ littermates, single-endpoint data sets were analyzed using a 2-way ANOVA (diet $\times$ genotype or surgery $\times$ genotype) with Tukey's HSD post hoc test. For repeated-measures endpoints, data were analyzed using a 2-way, repeated-measures ANOVA with Tukey's HSD post hoc test. Where appropriate, the Greenhouse-Geisser correction was applied for heterogeneity of variance. For all analyses, statistical significance was determined using GraphPad Prism, version 8.0 (GraphPad Software), with a $P$ value of less than 0.05 considered significant.

Study approval. All experiments followed NIH guidelines and were approved by the IACUC of the Medical College of Georgia.

\section{Author contributions}

AMS and DHG designed research studies, and DHG, MY, CMH, $\mathrm{HK}, \mathrm{BB}$, and AMS were directly involved in conducting experiments, acquiring data, analyzing data, and writing of the manuscript.

\section{Acknowledgments}

These studies were supported by grants from the NIH (K01DK100616, R03DK101817, R01DK110586, to AMS). We are grateful to Ari Waisman and Sergey Grivennikov for providing breeding pairs of IL-1R1-floxed mice and to Lin Mei for breeding pairs of Thy1-eGFP(S) mice. Experiments involving RNA extraction from paraffin-embedded samples were carried out with assistance from the Genomics Core facility at Augusta University.

Address correspondence to: Alexis M. Stranahan, Department of Neuroscience and Regenerative Medicine, Medical College of Georgia, Augusta University, 1120 15th Street, CA3009, Augusta, Georgia 30912, USA. Phone: 706.721.7885; Email: astranahan@ augusta.edu.

CMH's present address is: Department of Pharmaceutical Sciences, Appalachian College of Pharmacy, Oakwood, Virginia, USA. 
1. Xu W, Qiu C, Gatz M, Pedersen NL, Johansson B, Fratiglioni L. Mid- and late-life diabetes in relation to the risk of dementia: a population-based twin study. Diabetes. 2009;58(1):71-77.

2. $\mathrm{Xu} \mathrm{W}$, et al. Accelerated progression from mild cognitive impairment to dementia in people with diabetes. Diabetes. 2010;59(11):2928-2935.

3. Xu WL, Atti AR, Gatz M, Pedersen NL, Johansson B, Fratiglioni L. Midlife overweight and obesity increase late-life dementia risk: a population-based twin study. Neurology. 2011;76(18):1568-1574.

4. Whitmer RA, Gustafson DR, Barrett-Connor E, Haan MN, Gunderson EP, Yaffe K. Central obesity and increased risk of dementia more than three decades later. Neurology. 2008;71(14):1057-1064.

5. Sabia S, Kivimaki M, Shipley MJ, Marmot MG, Singh-Manoux A. Body mass index over the adult life course and cognition in late midlife: the Whitehall II Cohort Study. Am JClin Nutr. 2009;89(2):601-607.

6. Roriz-Cruz M, et al. Cognitive impairment and frontal-subcortical geriatric syndrome are associated with metabolic syndrome in a stroke-free population. Neurobiol Aging. 2007;28(11):1723-1736.

7. Wu W, et al. The brain in the age of old: the hippocampal formation is targeted differentially by diseases of late life. Ann Neurol. 2008;64(6):698-706.

8. Ho AJ, et al. Obesity is linked with lower brain volume in $700 \mathrm{AD}$ and MCI patients. Neurobiol Aging. 2010;31(8):1326-1339.

9. van den Berg E, Biessels GJ, de Craen AJ, Gussekloo J, Westendorp RG. The metabolic syndrome is associated with decelerated cognitive decline in the oldest old. Neurology. 2007;69(10):979-985

10. Sturman MT, de Leon CF, Bienias JL, Morris MC, Wilson RS, Evans DA. Body mass index and cognitive decline in a biracial community population. Neurology. 2008;70(5):360-367.

11. Xiong GL, Plassman BL, Helms MJ, Steffens DC. Vascular risk factors and cognitive decline among elderly male twins. Neurology. 2006;67(9):1586-1591.

12. Kanneganti TD, Dixit VD. Immunological complications of obesity. Nat Immunol. 2012;13(8):707-712.

13. Elias MF, Elias PK, Sullivan LM, Wolf PA, D'Agostino RB. Obesity, diabetes and cognitive deficit: The Framingham Heart Study. Neurobiol Aging. 2005;26 Suppl 1:11-16.

14. Kamogawa K, et al. Abdominal fat, adiposederived hormones and mild cognitive impairment: the J-SHIPP study. Dement Geriatr Cogn Disord. 2010;30(5):432-439.

15. Rosen ED, Spiegelman BM. What we talk about when we talk about fat. Cell. 2014;156(1-2):20-44.

16. Osborn O, Olefsky JM. The cellular and signaling networks linking the immune system and metabolism in disease. Nat Med. 2012;18(3):363-374.

17. Chawla A, Nguyen KD, Goh YP. Macrophage-mediated inflammation in metabolic disease. $\mathrm{Nat}$ Rev Immunol. 2011;11(11):738-749.

18. Vandanmagsar B, et al. The NLRP3 inflammasome instigates obesity-induced inflammation and insulin resistance. Nat Med. 2011;17(2):179-188.

19. Iyer SS, et al. Necrotic cells trigger a sterile inflammatory response through the Nlrp3 inflammasome. Proc Natl Acad Sci USA. 2009;106(48):20388-20393.

20. Youm YH, et al. Canonical Nlrp3 inflammasome links systemic low-grade inflammation to functional decline in aging. Cell Metab. 2013;18(4):519-532.

21. Heneka MT, et al. NLRP3 is activated in Alzheimer's disease and contributes to pathology in APP/PS1 mice. Nature. 2013;493(7434):674-678.

22. Banks WA, Kastin AJ. The interleukins-1 alpha, -1 beta, and -2 do not acutely disrupt the murine blood-brain barrier. Int JImmunopharmacol. 1992;14(4):629-636.

23. Weber A, Wasiliew P, Kracht M. Interleukin-1 (IL1) pathway. Sci Signal. 2010;3(105):cm1.

24. Sobesky JL, et al. Glucocorticoids mediate shortterm high-fat diet induction of neuroinflammatory priming, the NLRP3 inflammasome, and the danger signal HMGB1. eNeuro. 2016;3(4):ENEURO.0113-16.2016.

25. Salter MW, Stevens B. Microglia emerge as central players in brain disease. Nat Med. 2017;23(9):1018-1027.

26. Hao S, Dey A, Yu X, Stranahan AM. Dietary obesity reversibly induces synaptic stripping by microglia and impairs hippocampal plasticity. Brain Behav Immun. 2016;51:230-239.

27. Kanoski SE, Zhang Y, Zheng W, Davidson TL. The effects of a high-energy diet on hippocampal function and blood-brain barrier integrity in the rat. J Alzheimers Dis. 2010;21(1):207-219.

28. Yamamoto M, Guo DH, Hernandez CM, Stranahan AM. Endothelial Adora2a activation promotes blood-brain barrier breakdown and cognitive impairment in mice with diet-induced insulin resistance. J Neurosci. 2019;39(21):4179-4192.

29. Stranahan AM, Hao S, Dey A, Yu X, Baban B. Blood-brain barrier breakdown promotes macrophage infiltration and cognitive impairment in leptin receptor-deficient mice. JCereb Blood Flow Metab. 2016;36(12):2108-2121.

30. Buckman LB, et al. Obesity induced by a highfat diet is associated with increased immune cell entry into the central nervous system. Brain Behav Immun. 2014;35:33-42.

31. Zhang Y, et al. An RNA-sequencing transcriptome and splicing database of glia, neurons, and vascular cells of the cerebral cortex. J Neurosci. 2014;34(36):11929-11947.

32. Li Q, et al. Developmental heterogeneity of microglia and brain myeloid cells revealed by deep single-cell RNA sequencing. Neuron . 2019;101(2):207-223.e10.

33. Zhang CJ, et al. TLR-stimulated IRAKM activates caspase-8 inflammasome in microglia and promotes neuroinflammation. JClin Invest. 2018;128(12):5399-5412.

34. Bruttger J, et al. Genetic cell ablation reveals clusters of local self-renewing microglia in the mammalian central nervous system. Immunity. 2015;43(1):92-106.

35. Toda Y, Tsukada J, Misago M, Kominato Y, Auron PE, Tanaka Y. Autocrine induction of the human pro-IL-1beta gene promoter by IL-1beta in monocytes. JImmunol. 2002;168(4):1984-1991.

36. Parkhurst CN, et al. Microglia promote learning-dependent synapse formation through brain-derived neurotrophic factor. Cell.
2013;155(7):1596-1609.

37. Paré $\mathrm{A}$, et al. IL-1 $1 \beta$ enables CNS access to CCR $2^{\text {hi }}$ monocytes and the generation of pathogenic cells through GM-CSF released by CNS endothelial cells. Proc Natl Acad Sci USA. 2018;115(6):E1194-E1203.

38. Yona S, et al. Fate mapping reveals origins and dynamics of monocytes and tissue macrophages under homeostasis. Immunity. 2013;38(1):79-91.

39. Goldmann T, et al. A new type of microglia gene targeting shows TAK1 to be pivotal in CNS autoimmune inflammation. Nat Neurosci. 2013;16(11):1618-1626.

40. Goldmann T, et al. Origin, fate and dynamics of macrophages at central nervous system interfaces. Nat Immunol. 2016;17(7):797-805.

41. Pickering M, O'Connor JJ. Pro-inflammatory cytokines and their effects in the dentate gyrus. Prog Brain Res. 2007;163:339-354.

42. Chen Z, et al. Microglial displacement of inhibitory synapses provides neuroprotection in the adult brain. Nat Commun. 2014;5:4486.

43. Weinhard L, et al. Microglia remodel synapses by presynaptic trogocytosis and spine head filopodia induction. Nat Commun. 2018;9(1):1228.

44. Wen $\mathrm{H}$, et al. Fatty acid-induced NLRP3-ASC inflammasome activation interferes with insulin signaling. Nat Immunol. 2011;12(5):408-415.

45. Nagareddy PR, et al. Adipose tissue macrophages promote myelopoiesis and monocytosis in obesity. Cell Metab. 2014;19(5):821-835.

46. Konsman JP, Tridon V, Dantzer R. Diffusion and action of intracerebroventricularly injected interleukin-1 in the CNS. Neuroscience. 2000;101(4):957-967.

47. Derecki NC, et al. Regulation of learning and memory by meningeal immunity: a key role for IL-4. J Exp Med.2010;207(5):1067-1080.

48. Syková E. Extrasynaptic volume transmission and diffusion parameters of the extracellular space. Neuroscience. 2004;129(4):861-876.

49. Serrats J, Schiltz JC, García-Bueno B, van Rooijen N, Reyes TM, Sawchenko PE. Dual roles for perivascular macrophages in immune-to-brain signaling. Neuron. 2010;65(1):94-106

50. Audoy-Rémus J, Richard JF, Soulet D, Zhou H, Kubes P, Vallières L. Rod-shaped monocytes patrol the brain vasculature and give rise to perivascular macrophages under the influence of proinflammatory cytokines and angiopoietin-2. JNeurosci. 2008;28(41):10187-10199.

51. Cunningham ET, Wada E, Carter DB, Tracey DE, Battey JF, De Souza EB. In situ histochemical localization of type I interleukin-1 receptor messenger RNA in the central nervous system, pituitary, and adrenal gland of the mouse. J Neurosci. 1992;12(3):1101-1114

52. Srinivasan D, Yen JH, Joseph DJ, Friedman W. Cell type-specific interleukin-1beta signaling in the CNS. J Neurosci. 2004;24(29):6482-6488.

53. Liu X, et al. Interleukin 1 type 1 receptor restore: a genetic mouse model for studying interleukin receptor-mediated effects in specific cell types. J Neurosci. 2015;35(7):2860-2870.

54. Huang Y, Smith DE, Ibáñez-Sandoval O, Sims JE, Friedman WJ. Neuron-specific effects of interleukin- $1 \beta$ are mediated by a novel isoform of the IL-1 receptor accessory protein. J Neurosci. 
2011;31(49):18048-18059.

55 . Smith DE, et al. A central nervous system-restricted isoform of the interleukin-1 receptor accessory protein modulates neuronal responses to interleukin-1. Immunity. 2009;30(6):817-831.

56. Prieto GA, et al. Synapse-specific IL-1 receptor subunit reconfiguration augments vulnerability to IL-1 $\beta$ in the aged hippocampus. Proc Natl Acad Sci USA. 2015;112(36):E5078-E5087.

57. Katsuki H, Nakai S, Hirai Y, Akaji K, Kiso Y, Satoh M. Interleukin-1 beta inhibits long-term potentiation in the CA3 region of mouse hippocampal slices. Eur J Pharmacol. 1990;181(3):323-326.

58. Kelly A, et al. The anti-inflammatory cytokine, interleukin (IL)-10, blocks the inhibitory effect of IL-1 beta on long term potentiation. A role for JNK. J Biol Chem. 2001;276(49):45564-45572.

59. Lynch MA. Neuroinflammatory changes negatively impact on LTP: A focus on IL-1 $\beta$. Brain Res. 2015;1621:197-204.

60. Vereker E, O'Donnell E, Lynch MA. The inhibitory effect of interleukin-1beta on long-term potentiation is coupled with increased activity of stress-activated protein kinases. J Neurosci. 2000;20(18):6811-6819.

61. Avital A, et al. Impaired interleukin-1 signaling is associated with deficits in hippocampal memory processes and neural plasticity. Hippocampus. 2003;13(7):826-834.

62. Biancardi VC, Stranahan AM, Krause EG, de Kloet AD, Stern JE. Cross talk between AT1 receptors and Toll-like receptor 4 in microglia contributes to angiotensin II-derived ROS production in the hypothalamic paraventricular nucleus. Am J Physiol Heart Circ Physiol. 2016;310(3):H404-H415.
63. Wang Q, Rowan MJ, Anwyl R. Beta-amyloid-mediated inhibition of NMDA receptor-dependent long-term potentiation induction involves activation of microglia and stimulation of inducible nitric oxide synthase and superoxide. J Neurosci. 2004;24(27):6049-6056.

64. Wosiski-Kuhn M, Erion JR, Gomez-Sanchez EP, Gomez-Sanchez CE, Stranahan AM. Glucocorticoid receptor activation impairs hippocampal plasticity by suppressing BDNF expression in obese mice. Psychoneuroendocrinology. 2014;42:165-177.

65. Fotuhi M, Hachinski V, Whitehouse PJ. Changing perspectives regarding late-life dementia. Nat Rev Neurol. 2009;5(12):649-658.

66. Bos I, et al. The frequency and influence of dementia risk factors in prodromal Alzheimer's disease. Neurobiol Aging. 2017;56:33-40.

67. Gu Y, et al. Mediterranean diet and brain structure in a multiethnic elderly cohort. Neurology. 2015;85(20):1744-1751.

68. Hillman CH, Erickson KI, Kramer AF. Be smart, exercise your heart: exercise effects on brain and cognition. Nat Rev Neurosci. 2008;9(1):58-65.

69. Handley JD, Williams DM, Caplin S, Stephens JW, Barry J. Changes in cognitive function following bariatric surgery: a systematic review. Obes Surg. 2016;26(10):2530-2537.

70. Marques EL, et al. Changes in neuropsychological tests and brain metabolism after bariatric surgery. J Clin Endocrinol Metab. 2014;99(11):E2347-E2352.

71. Hawkins MA, et al. The association between reduced inflammation and cognitive gains after bariatric surgery. Psychosom Med. 2015;77(6):688-696.
72. Fabbrini E, et al. Surgical removal of omental fat does not improve insulin sensitivity and cardiovascular risk factors in obese adults. Gastroenter ology. 2010;139(2):448-455.

73. Dunn JP, et al. Hepatic and peripheral insulin sensitivity and diabetes remission at 1 month after Roux-en-Y gastric bypass surgery in patients randomized to omentectomy. Diabetes Care. 2012;35(1):137-142.

74. Lee Y, Pędziwiatr M, Major P, Brar K, Doumouras AG, Hong D. The effect of omentectomy added to bariatric surgery on metabolic outcomes: a systematic review and meta-analysis of randomized controlled trials. Surg Obes Relat Dis. 2018;14(11):1766-1782.

75. Erion JR, et al. Obesity elicits interleukin 1-mediated deficits in hippocampal synaptic plasticity. J Neurosci. 2014;34(7):2618-2631.

76. Stranahan AM, Arumugam TV, Cutler RG, Lee K, Egan JM, Mattson MP. Diabetes impairs hippocampal function through glucocorticoidmediated effects on new and mature neurons. Nat Neurosci. 2008;11(3):309-317.

77. Stranahan AM, et al. Hippocampal gene expression patterns underlying the enhancement of memory by running in aged mice. Neurobiol Aging. 2010;31(11):1937-1949.

78. Banks WA, Ortiz L, Plotkin SR, Kastin AJ. Human interleukin (IL) 1 alpha, murine IL-1 alpha and murine IL-1 beta are transported from blood to brain in the mouse by a shared saturable mechanism. J Pharmacol Exp Ther. 1991;259(3):988-996.

79. Dey A, Hao S, Erion JR, Wosiski-Kuhn M, Stranahan AM. Glucocorticoid sensitization of microglia in a genetic mouse model of obesity and diabetes. J Neuroimmunol. 2014;269(1-2):20-27. 